\title{
Revisiting the two-dimensional defect-free azimuthal nematic equilibrium on an annulus.
}

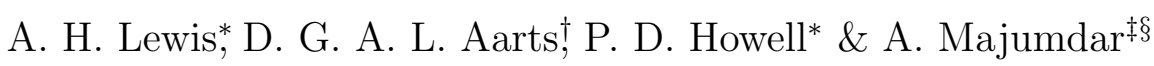

August 19, 2017

\begin{abstract}
We study the azimuthal defect-free nematic state on a two-dimensional annulus within a simplified and reduced two-dimensional Landau-de Gennes model for nematic liquid crystals. We perform a detailed asymptotic analysis of the instabilities of the defect-free state in terms of a dimensionless material and temperature-dependent variable and the annular aspect ratio. The asymptotic analysis is accompanied by a rigorous local stability result, again in terms of a dimensionless material and temperature-dependent parameter and annular aspect ratio. In contrast to Oseen-Frank predictions, the defect-free state can be unstable in this model, with elastic isotropy and strong anchoring, for a range of macroscopically relevant annular aspect ratios.
\end{abstract}

\section{Introduction}

Nematic liquid crystals (LCs) are classic examples of complex liquids with a certain degree of long-range orientational ordering i.e. the constituent molecules typically align along some locally preferred directions leading to partial ordering $[15,40]$. Nematics in confinement exhibit complex spatio-temporal patterns, often with topological defects and a lot remains to be understood about how to predict, control and tune nematic pattern formation tailored to specific experiments or applications. We re-visit the classical problem of a nematic sample in a two-dimensional (2D) annulus with strong tangential boundary conditions and no external fields. The tangential boundary conditions imply that on the circular boundaries, the nematic molecules preferentially follow the circular boundaries and this model framework is motivated in part by recent experiments on rod-like $f d$-viruses in shallow, annular microscopic chambers $[1,13,17]$ for which the experimentalists observe multiple (meta-)stable states, including a radially-invariant defect-free state and states with regularly arranged defects on the boundary; see Figure 1.

In this paper, we focus on the radially-invariant defect-free state on an annulus, often referred to as the azimuthal state in the literature; see Figure 1(a). This model problem is well-studied in the literature, especially within the Oseen-Frank (OF) theory for liquid crystals which assumes that the confined nematic sample has a single distinguished direction

\footnotetext{
${ }^{*}$ Mathematical Institute, University of Oxford, Andrew Wiles Building, Oxford OX2 6GG, UK

${ }^{\dagger}$ Department of Chemistry, University of Oxford, Physical and Theoretical Chemistry Laboratory, Oxford OX1 3QZ, UK

${ }^{\ddagger}$ Department of Mathematical Sciences, University of Bath, Bath BA2 7AY, UK

${ }^{\S}$ Corresponding author: a.majumdar@bath.ac.uk
} 
of preferred molecular alignment with a constant degree of orientational ordering. We do not perform an exhaustive literature review here for sake of brevity but refer the reader to the papers $[3-5,7,9,10,37,38]$ that focus on nematic equilibria within annular chambers. These papers focus on the stability and multiplicity of nematic equilibria on 2D annuli, or between coaxial cylinders with a combination of weak and strong boundary conditions, different kinds of boundary conditions (homeotropic or radial, azimuthal or tangential, uniform etc.), including the effects of external fields and, in some cases, higher-dimensional biaxial effects. In [7], the authors rigorously demonstrate the instability of the defect-free state for wide annuli, with respect to out-of-plane perturbations.

We focus on a specific problem in this paper, largely building on our recent work in [27]. In [27], we model planar nematic equilibria on a $2 \mathrm{D}$ annulus with tangential boundary conditions, within the Oseen-Frank theory, looking at the azimuthal defect-free state and nematic configurations with equally spaced boundary defects, in light of the recent experiments in $[1,13]$; also see [33] for related theoretical work. In [27], we have three key model variables: the elastic anisotropy $\delta$ in terms of the Oseen-Frank elastic constants, the annular aspect ratio $\rho$, and the anchoring strength $\alpha$ which is a measure of how strongly the tangent boundary conditions are implemented on the circular boundaries. We study the stability of the azimuthal defect-free state in terms of $\delta, \rho$ and $\alpha$ and, in particular, we find that this simple state is always stable in the one-constant model $(\delta=0)$ with strong anchoring or Dirichlet boundary conditions ( $\alpha \rightarrow \infty$ limit) for macroscopically relevant values of $\rho$. When comparing the Oseen-Frank energies of the azimuthal defect-free state and those of configurations with equally spaced boundary defects, we find that the azimuthal defect-free state is almost always energetically preferable to configurations with boundary defects unless we have large elastic anisotropy $(\delta \rightarrow 1)$ or relatively weak to moderate anchoring (smaller values of $\alpha$ ).

However, experiments show that the azimuthal (or equivalently radially invariant) defectfree state may be unstable for particular systems with $\delta \approx 0$ and relatively strong anchoring. We do not have enough data to make quantitative comparisons between the phase diagrams in [27] and the experiments in $[1,13]$, but it is possible that the simplified Oseen-Frank model in [27] does not capture all the instabilities of the azimuthal defect-free state. In this paper, we therefore analyze the same azimuthal defect-free state using a more sophisticated Landaude Gennes (LdG) theory. We adopt a reduced two-dimensional LdG approach and model the nematic state in terms of a reduced LdG order parameter: a 2D LdG order parameter represented by a symmetric, traceless $2 \times 2$ matrix with just two degrees of freedom. In some sense, this is just a weighted Oseen-Frank model with a planar nematic director $\mathbf{n}$ that models the single distinguished direction of molecular alignment in the plane, and a scalar order parameter $s$ that measures the degree of alignment about $\mathbf{n}$. This $2 \mathrm{D}$ LdG approach has been used to model planar nematic equilibria on $2 \mathrm{D}$ geometries in $[24,28,39]$ and is advantageous compared to the Oseen-Frank approach because (i) it contains information about $s$ and (ii) the 2D LdG energy density comprises the one-constant Dirichlet energy density and a 2D LdG bulk potential that contains information about the temperature and imposes a preferred bulk value of $s$. We work with Dirichlet tangential boundary conditions on the circular boundaries of the annulus and study the stability/instability of the defect-free state in terms of the annular aspect ratio $\rho$ and a dimensionless material and temperaturedependent parameter, denoted by $\lambda$.

We have two main results: a rigorous local stability result in terms of an explicit relation between $\lambda$ and $\rho$ followed by a detailed asymptotic analysis that delineates the regimes of stability and instability in the $(\lambda, \rho)$-parameter space. In particular, we identify a critical 
curve in the $(\lambda, \rho)$-plane and compute its asymptotic behaviour as $\rho \rightarrow 0$ and as $\lambda \rightarrow \infty$. The asymptotic analysis yields quantitative estimates, and predicts that, for sufficiently small $\rho$, the defect-free state is unstable for a range of values of $\lambda$ i.e. for $\lambda_{\min }(\rho)<\lambda<\lambda_{\max }(\rho)$. For larger values of $\rho$, the defect-free state is locally stable for all $\lambda$. The asymptotic analysis is corroborated by some numerical experiments on the gradient-flow model for the $2 \mathrm{D}$ LdG energy to study the dynamic evolution of states with defect-free initial conditions. For $\lambda_{\min }(\rho)<\lambda<\lambda_{\max }(\rho)$, the defect-free initial condition evolves to a state with two interior point defects on the annulus, and these defects relax and disappear as $\lambda$ increases past $\lambda_{\max }(\rho)$ or decreases past $\lambda_{\min }(\rho)$. Interestingly, this analysis in a 2D LdG framework shows that the azimuthal defect-free state can be unstable with elastic isotropy and strong anchoring, for a range of macroscopically relevant values of $\rho$, in contrast to the simplified Oseen-Frank predictions in [27].

Finally, we relate our results in the restricted $2 \mathrm{D}$ LdG framework to exact equilibria in the fully three-dimensional (3D) LdG framework. We do this in terms of the radially symmetric LdG solutions, that are exact solutions of the Euler-Lagrange equations associated with a one-constant version of the 3D LdG energy on coaxial concentric cylinders, introduced in [16] and analyzed in detail in [22]. In particular, the stability/instability of these solutions as a function of their winding number or degree, has been analyzed carefully in [20,21]. Our results are not subsumed by the results in [16,20-22], particularly since these papers focus on a disk and we have a geometrical parameter, $\rho$, which in turn implies that we lose the crucial monotonicity properties exploited in these previous works. Further, our asymptotic estimates remain of independent interest. We can relate our $2 \mathrm{D}$ defect-free state in the 2D LdG framework to a radially symmetric critical point of the full 3D LdG energy on an annulus with appropriately prescribed Dirichlet conditions, at a special temperature. The local stability result in the 2D framework only gives insight into stability with respect to a restricted class of perturbations (to be made precise later) and not all admissible perturbations. The instability results carry over to the $3 \mathrm{D}$ framework i.e. if the $2 \mathrm{D}$ defect-free state is unstable in the 2D LdG framework, then the corresponding 3D radially symmetric solution is also unstable in the 3D LdG framework, although the parameter regime of instability is larger in the 3D framework compared to the 2D framework. We also make the connection to 3D problems using $\Gamma$-convergence results (e.g. thin film results) established in [19] which show that minimizers of the 3D LdG energy on particular "thin" 3D domains with particular boundary conditions, will necessarily have a 2D character in certain asymptotic limits. Finally, our asymptotic and numerical results yield interesting quantitative estimates for the stability of the defect-free state, and such quantitative estimates are amenable to experimental tests.

The paper is organized as follows. In Section 2, we review the modelling framework. In Section 3, we define the 2D azimuthal defect-free state on an annulus with Dirichlet tangent boundary conditions, compute the rigorous local stability result (following the recipe by [34] in the Ginzburg-Landau setting) and perform the detailed asymptotic analysis. In Section 4, we relate our $2 \mathrm{D}$ results to a radially symmetric critical point of a 3D LdG energy, as introduced in [16] and conclude in Section 5 with some outlooks and perspectives.

\section{Theory and modelling}

The Landau-de Gennes ( $\mathrm{LdG}$ ) theory is one of the most general continuum theories for nematic LCs to date $[15,36]$. The LdG theory describes the nematic state by an order 


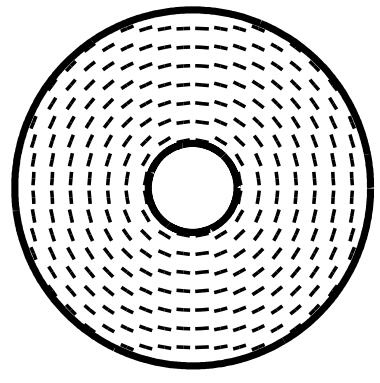

(a)

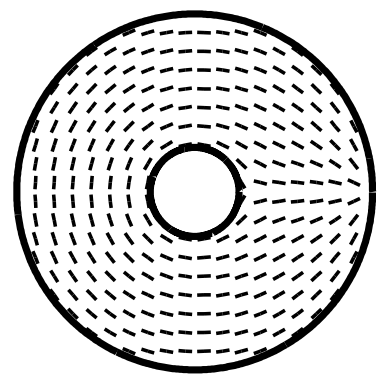

(b)

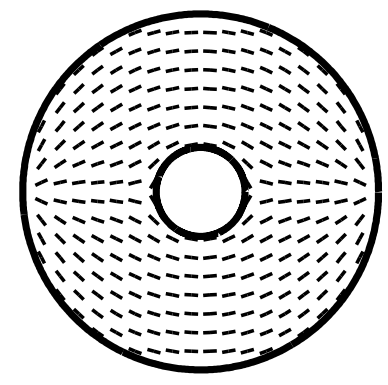

(c)

Figure 1: Some of the nematic director fields identified experimentally in $[1,13,17]$. Reproduced from [27] with permission.

parameter, the LdG Q-tensor, that is a macroscopic measure of the LC anisotropy. For threedimensional (3D) problems, the LdG Q-tensor is a symmetric, traceless $3 \times 3$ matrix, with five degrees of freedom $[15,36]$. A nematic phase is said to be (i) isotropic if $\mathbf{Q}=0$, (ii) uniaxial if $\mathbf{Q}$ has two degenerate non-zero eigenvalues with a single distinguished eigenvector and (iii) biaxial if $\mathbf{Q}$ has three distinct eigenvalues.

The experimentally observed states for LC confined to a domain $\Omega$ are typically modelled in terms of either critical points or local/global minimizers of an appropriately defined LdG energy $[2,36]$. A prototypical LdG energy functional is given by

$$
I[\mathbf{Q}]:=\int_{\Omega} w(\mathbf{Q}, \nabla \mathbf{Q})+f_{B}(\mathbf{Q}) \mathrm{d} \Omega,
$$

where $w$ is an elastic energy density that penalizes spatial inhomogeneities and $f_{B}$ is a bulk potential that drives the nematic-isotropic phase transition as a function of the temperature or concentration. We work with the widely used one-constant elastic energy density (corresponding to elastic isotropy) and quartic bulk energy density, namely

$$
w(\mathbf{Q}, \nabla \mathbf{Q})=\frac{L}{2}|\nabla \mathbf{Q}|^{2}, \quad f_{B}(\mathbf{Q})=\frac{A}{2} \operatorname{tr} \mathbf{Q}^{2}-\frac{B}{3} \operatorname{tr} \mathbf{Q}^{3}+\frac{C}{4}\left(\operatorname{tr} \mathbf{Q}^{2}\right)^{2},
$$

where $L, A, B, C$ are material parameters. Usually, $L, B$ and $C$ are taken to be constant and positive, while $A$ varies linearly with temperature. In this paper we are concerned with the regime $A<0$, for which the isotropic state $\mathbf{Q}=0$ is an unstable critical point of $f_{B}$ and $f_{B}$ attains its minimum at an ordered nematic state, namely

$$
\mathbf{Q}_{\text {min }}=s_{+}\left(\mathbf{n} \otimes \mathbf{n}-\frac{\mathbf{I}}{3}\right) ; \quad s_{+}=\frac{B+\sqrt{B^{2}-24 A C}}{4 C},
$$

with $\mathbf{n}$ an arbitrary unit vector. The critical points of the LdG energy in (1) and (2) are classical solutions of the associated Euler-Lagrange equations [29, 32]:

$$
L \Delta \mathbf{Q}_{i j}=A \mathbf{Q}_{i j}-B\left(\mathbf{Q}_{i p} \mathbf{Q}_{p j}-\operatorname{tr} \mathbf{Q}^{2} \frac{\delta_{i j}}{3}\right)+C|\mathbf{Q}|^{2} \mathbf{Q}_{i j} ; \quad i, j=1,2,3
$$


and (4) is in general a system of five elliptic coupled partial differential equations. There are few semi-explicit examples of exact solutions of (4), some notable ones being the radialhedgehog solution, the radially-symmetric $(u, v)$ solutions and the well order reconstruction solution $[12,16,23,35]$.

Our domain is a two-dimensional annulus defined by

$$
\Omega=\left\{(r, \phi) \in \mathbb{R}^{2}: \quad R_{\text {in }} \leq r \leq R_{\text {out }}, \quad 0 \leq \phi<2 \pi\right\},
$$

using plane polar coordinates. We adopt a simplified 2D LdG framework for which the nematic state is described by a symmetric traceless $2 \times 2$ matrix of the form

$$
\mathbf{Q}=s\left(2 \mathbf{n} \otimes \mathbf{n}-\mathbf{I}_{2}\right),
$$

where $s$ is a real-valued scalar order parameter, $\mathbf{n}$ is a two-dimensional unit-vector and $\mathbf{I}_{2}$ is the $2 \times 2$ identity matrix [30]. It is clear from (6) that a 2D Q-tensor only has two degrees of freedom: the order parameter, $s(r, \phi)$, that is a measure of the degree of the orientational order, and the director field, $\mathbf{n}(r, \phi) \in S^{1}$, that represents the distinguished direction of nematic alignment. We recall that the Oseen-Frank theory describes the nematic state by the director, $\mathbf{n}$, and the 2D LdG model therefore contains more information than the OseenFrank approach but considerably less information than the 3D LdG model desribed above. In particular, a 2D LdG Q-tensor cannot describe biaxiality or three-dimensional director fields.

For $\mathbf{Q}$ as in (6), $\operatorname{tr} \mathbf{Q}^{3}=0$, and hence the $\mathrm{LdG}$ energy (1) reduces to

$$
I[\mathbf{Q}]=\int_{\Omega} \frac{L}{2}|\nabla \mathbf{Q}|^{2}+\frac{A}{2}|\mathbf{Q}|^{2}+\frac{C}{4}|\mathbf{Q}|^{4} \mathrm{~d} \Omega,
$$

where $|\mathbf{Q}|^{2}=\operatorname{tr} \mathbf{Q}^{2}=2 s^{2}$. By scaling $\mathbf{Q}$ with $\sqrt{|A| / C}$ and $r$ with $R_{\text {out }}$, the LdG energy in (7) may be normalized to the Ginzburg-Landau functional in superconductivity [8,30], namely

$$
I[\mathbf{Q}]=\int_{\Omega} \frac{1}{2}|\nabla \mathbf{Q}|^{2}+\frac{\lambda}{4}\left(|\mathbf{Q}|^{2}-1\right)^{2} \mathrm{~d} \Omega,
$$

where

$$
\lambda=|A| R_{\text {out }}^{2} / L,
$$

and $\Omega$ is now the rescaled annulus $\rho \leq r \leq 1$ and

$$
\rho=R_{\text {in }} / R_{\text {out }} .
$$

We can physically think of $\lambda$ as being a dimensionless parameter that is the square of the ratio of a geometric length scale, $R_{\text {out }}$ to the uniaxial correlation length, $\xi^{2}=L /|A|$ i.e. large $\lambda$ corresponds to a macroscopic domain, for example if $R_{\text {out }} \sim 10^{-6} \mathrm{~m},|A| \sim 10^{5} \mathrm{~N} / \mathrm{m}^{2}$ and $L=10^{-11} N$ [15], then $\lambda \sim 10^{10}$.

The Euler-Lagrange equations corresponding to (8) are simply the Ginzburg-Landau equations [8]:

$$
\Delta \mathbf{Q}_{i j}=\lambda \mathbf{Q}_{i j}\left(|\mathbf{Q}|^{2}-1\right)=\lambda \mathbf{Q}_{i j}\left(2 s^{2}-1\right) ; \quad i, j=1,2 .
$$

Spatially uniform solutions of (9) consist of the unstable isotropic equilibrium $\mathbf{Q}=0$ and stable uniaxial equilibria with $s=1 / \sqrt{2}$.

We work with tangent boundary conditions which imply that $\mathbf{n}$ must follow the inner and outer circular boundaries. We also assume that $s=1 / \sqrt{2}$ on $\partial \Omega$, implying that the nematic 
phase is maximally ordered at the boundaries. In the $2 \mathrm{D} \mathrm{LdG}$ framework, we therefore impose the Dirichlet condition $\mathbf{Q}=\mathbf{Q}_{b}$ on $r=\rho$, 1 , where

$$
\mathbf{Q}_{b}=\frac{1}{\sqrt{2}}\left(2 \mathbf{e} \otimes \mathbf{e}-\mathbf{I}_{2}\right),
$$

and $\mathbf{e}=(-\sin \phi, \cos \phi)$ is a unit-vector along each circular boundary . As with any problem in the calculus of variations, we are interested in the critical points of the LdG energy (8) subject to this Dirichlet boundary condition, and in their stability in terms of the geometrical parameter $\rho$ and the material parameter $\lambda$. In the next section, we define the defect-free state in this $2 \mathrm{D}$ LdG framework and perform the parallel stability analysis.

\section{The defect-free state in the 2D LdG framework}

\subsection{Definition}

The azimuthal defect-free state is a "bend" state that follows the circular boundaries everywhere inside the annular domain $\Omega$ defined in Section 2. In the 2D LdG framework, it is described by a matrix of the form (6) that is a solution of the two-dimensional system (9). We define the defect-free state in the 2D LdG framework to be a solution of (9) of the form

$$
\mathbf{Q}^{*}=s(r)\left(2 \mathbf{e} \otimes \mathbf{e}-\mathbf{I}_{2}\right),
$$

where $s:[\rho, 1] \rightarrow \mathbb{R}$ is the unknown scalar order parameter (determined by (9)) subject to the Dirichlet conditions

$$
s(\rho)=s(1)=\frac{1}{\sqrt{2}} .
$$

The LdG energy of $\mathbf{Q}^{*}$ is computed using (8) to be

$$
I\left[\mathbf{Q}^{*}\right]=2 \pi \int_{\rho}^{1}\left(\left(s^{\prime}\right)^{2}+\frac{4 s^{2}}{r^{2}}+\frac{\lambda}{4}\left(2 s^{2}-1\right)^{2}\right) r \mathrm{~d} r
$$

and we define the optimal order parameter $s$ in (11) to be a minimizer of (13) subject to the Dirichlet conditions (12). It is straightforward to verify (see $[25,31]$ ) that the minimizing $s$ is a classical non-negative solution of the second-order ordinary differential equation

$$
s^{\prime \prime}+\frac{s^{\prime}}{r}-\frac{4 s}{r^{2}}=\lambda s\left(2 s^{2}-1\right),
$$

subject to the boundary conditions (12), and that $\mathbf{Q}^{*}$, thus defined in terms of the optimal $s$ in (13)-(14), is indeed a critical point of the reduced LdG energy (8). We note that this is equivalent to the familiar radial problem where $\mathbf{Q}_{b}=\frac{1}{\sqrt{2}}\left(2 \mathbf{e}_{r} \otimes \mathbf{e}_{r}-\mathbf{I}_{2}\right)$ and $\mathbf{e}_{r}=(\cos \phi, \sin \phi)$ is the radial unit-vector; the analogous defect-free state in this case would be of the form (11) with $\mathbf{e}$ being replaced by $\mathbf{e}_{r}$.

\subsection{Rigorous stability analysis}

In this section, we prove that the defect-free state in (11) is locally stable, at least for certain ranges of the parameters $\lambda$ and $\rho$. 
Proposition 1. The defect-free state, $\mathbf{Q}^{*}$, defined in (11)-(14), is a locally stable equilibrium of the reduced $L d G$ energy (8) for all $0<\rho<1$ provided

$$
\begin{aligned}
\text { either } \quad \lambda & >\frac{3\left(1+\rho^{2}\right)^{2}}{2 \rho^{4}}, \\
\text { or } \quad \lambda & <\frac{\pi^{2}\left(1+\rho^{2}\right)^{2}}{\left(1-\rho^{2}\right)^{2}(\log \rho)^{2}},
\end{aligned}
$$

or both; see Figure 2.

Proof. We follow the same strategy as in [34], where the author establishes the local stability of the defect-free state on a disc in two dimensions, as opposed to an annulus. For a disc, the corresponding order parameter is defined for $0 \leq r \leq 1$, vanishes at $r=0$ and is a monotonically increasing function subject to a Dirichlet condition at $r=1$. The function $s$ satisfying (12) and (14) cannot be a monotone function; by application of the maximum principle we must have $s^{2}(r) \leq 1 / 2$ for $\rho \leq r \leq 1$, and hence $s$ must have an intermediate local minimum [31]. In fact, the function $s$ is strictly positive and one can show that

$$
s(r) \geq \frac{\sqrt{2} \rho}{\rho^{2}+1}
$$

for all $0<\rho<r<1$ (see [18]), independently of the value of $\lambda$.

The second variation of the LdG energy (8) about $\mathbf{Q}^{*}$ is given by

$$
\delta^{2} I\left[\mathbf{Q}^{*}\right]:=\int_{\Omega} \frac{1}{2}|\nabla \mathbf{V}|^{2}+\frac{\lambda}{2}\left(2 s^{2}-1\right)|\mathbf{V}|^{2}+\lambda\left(\mathbf{Q}^{*} \cdot \mathbf{V}\right)^{2} \mathrm{~d} \Omega,
$$

where $\mathbf{V}(r, \phi) \in S_{2}$ is an admissible perturbation about $\mathbf{Q}^{*}$ with $\mathbf{V}(\rho, \phi)=\mathbf{V}(1, \phi)=0$. We use the following basis for the space of symmetric, traceless $2 \times 2$ matrices:

$$
\mathbf{E}=\left(\begin{array}{cc}
\cos (2 \phi) & \sin (2 \phi) \\
\sin (2 \phi) & -\cos (2 \phi)
\end{array}\right), \quad \quad \mathbf{F}=\left(\begin{array}{cc}
-\sin (2 \phi) & \cos (2 \phi) \\
\cos (2 \phi) & \sin (2 \phi)
\end{array}\right)
$$

Equivalently, $\mathbf{E}=2 \mathbf{e}_{r} \otimes \mathbf{e}_{r}-I_{2}$ and $\mathbf{F}=\mathbf{e}_{r} \otimes \mathbf{e}+\mathbf{e} \otimes \mathbf{e}_{r}$, where $\mathbf{e}_{r}=(\cos \phi, \sin \phi)$ and $\mathbf{e}=(-\sin \phi, \cos \phi)$ are the radial and azimuthal $2 \mathrm{D}$ unit vectors introduced above. Then, an arbitrary $\mathbf{V}$ can be written as

$$
\mathbf{V}=v(r, \phi) \mathbf{E}+w(r, \phi) \mathbf{F},
$$

where $v(\rho, \phi)=v(1, \phi)=w(\rho, \phi)=w(1, \phi)=0$ and $v, w$ are $2 \pi$-periodic in $\phi$. With $\mathbf{Q}^{*}$ given by (11) and perturbations of the form (19), the second variation (17) is given by

$$
\begin{aligned}
\delta^{2} I\left[\mathbf{Q}^{*}\right]=\int_{\Omega}\left[|\nabla v|^{2}+|\nabla w|^{2}+\frac{4}{r^{2}}\left(v^{2}+w^{2}\right.\right. & \left.+v \frac{\partial w}{\partial \phi}-w \frac{\partial v}{\partial \phi}\right) \\
& \left.+\lambda\left(6 s^{2}-1\right) v^{2}+\lambda\left(2 s^{2}-1\right) w^{2}\right] \mathrm{d} \Omega .
\end{aligned}
$$

We use a Fourier decomposition for the functions, $v$ and $w$, that is,

$$
v(r, \phi)=\sum_{n=0}^{\infty} a_{n}(r) \cos (n \phi)+b_{n}(r) \sin (n \phi), \quad w(r, \phi)=\sum_{n=0}^{\infty} c_{n}(r) \cos (n \phi)+d_{n}(r) \sin (n \phi),
$$


where $a_{n}=b_{n}=c_{n}=d_{n}=0$ on $r=\rho, 1$ for all $n$. The second variation (20) can thus be expressed as

$$
\delta^{2} I\left[\mathbf{Q}^{*}\right]=2 \pi L_{0}\left[a_{0}, c_{0}\right]+\pi \sum_{n=1}^{\infty}\left(L_{n}\left[a_{n},-d_{n}\right]+L_{n}\left[b_{n}, c_{n}\right]\right),
$$

where the functionals $L_{n}$ are given by

$$
\begin{aligned}
L_{n}[b, c]:=\int_{\rho}^{1}\left(\left(b^{\prime}\right)^{2}+\left(c^{\prime}\right)^{2}+\frac{n^{2}+4}{r^{2}}\left(b^{2}+c^{2}\right)\right. & -\frac{8 n}{r^{2}} b c \\
& \left.+\lambda\left(6 s^{2}-1\right) b^{2}+\lambda\left(2 s^{2}-1\right) c^{2}\right) r \mathrm{~d} r .
\end{aligned}
$$

The key ingredient in the proof of Proposition 1 is to show that $\min L_{n}[b, c]>0$ for non-trivial $b, c$.

Remark. We first note that $\delta^{2} I\left[\mathbf{Q}^{*}\right]>0$ if $\min L_{0}>0, \min L_{1}>0$ and $\min L_{2}>0$, and hence we need only focus on $L_{0}, L_{1}, L_{2}$ in the stability analysis. This can be checked easily by computing

$$
L_{n+2}[b, c]-L_{n}[b, c]=4 \int_{\rho}^{1}\left[(n-1)\left(b^{2}+c^{2}\right)+2(b-c)^{2}\right] \frac{\mathrm{d} r}{r} \geq 0
$$

for $n \geq 1$.

The case $n=0$. The integral for $n=0$ satisfies

$$
L_{0}\left[a_{0}, c_{0}\right] \geq I_{0}\left[a_{0}\right]+I_{0}\left[c_{0}\right],
$$

where

$$
I_{0}[a]=\int_{\rho}^{1}\left[\left(a^{\prime}\right)^{2}+\frac{4}{r^{2}} a^{2}+\lambda\left(2 s^{2}-1\right) a^{2}\right] r \mathrm{~d} r .
$$

We let $a(r)=s(r) \alpha(r)$ and recall the governing ordinary differential equation (14) for $s$ to obtain

$$
I_{0}[s \alpha]=\int_{\rho}^{1}\left(r \alpha^{2} s s^{\prime}\right)^{\prime}+r s^{2}\left(\alpha^{\prime}\right)^{2} \mathrm{~d} r=\int_{\rho}^{1} r s^{2}\left(\alpha^{\prime}\right)^{2} \mathrm{~d} r>0
$$

for any non-trivial $\alpha:[\rho, 1] \rightarrow \mathbb{R}$ with $\alpha(\rho)=\alpha(1)=0$.

The case $n=1$. To analyse the case where $n=1$, we let $s=u$ be the minimizer of the functional (13), subject to the modified boundary conditions

$$
u(\rho)=0, \quad u(1)=\frac{1}{\sqrt{2}} .
$$

Such a minimizer, $u$, is guaranteed to exist from direct methods in the calculus of variations, and is a classical solution of

$$
u^{\prime \prime}+\frac{u^{\prime}}{r}-\frac{4 u}{r^{2}}=\lambda\left(2 u^{2}-1\right) u
$$

satisfying the boundary conditions (28). 
Lemma 1. The function $u$ is unique, monotonically increasing and $u \leq s$ for all $r$.

Proof. One can show that $u$ is unique and monotonically increasing by an immediate adaptation of the arguments in [25], which are omitted here for brevity. We show that $u \leq s$. Suppose for a contradiction that $s(r)<u(r)$ for some $r \in(\rho, 1)$. Then, there must exist $r_{1}$ and $r_{2}$, where $\rho<r_{1}<r_{2} \leq 1$, for which $s\left(r_{1}\right)-u\left(r_{1}\right)=0, s^{\prime}\left(r_{1}\right)-u^{\prime}\left(r_{1}\right)<0, s\left(r_{2}\right)-u\left(r_{2}\right)=0$, $s^{\prime}\left(r_{2}\right)-u^{\prime}\left(r_{2}\right)>0$ and $s(r)-u(r)<0$ for $r \in\left[r_{1}, r_{2}\right]$. We multiply equations (14) and (29) for $s$ and $u$ by $r u$ and $r s$ respectively, subtract and integrate over $\left(r_{1}, r_{2}\right)$ to find

$$
\left[r u s^{\prime}-r s u^{\prime}\right]_{r_{1}}^{r_{2}}=\int_{r_{1}}^{r_{2}} 2 \lambda u s\left(s^{2}-u^{2}\right) r \mathrm{~d} r .
$$

The left-hand side is positive since $s=u$ at $r=r_{1}$ and $r=r_{2}$, and the right-hand side is negative since $s-u<0$ for $r \in\left(r_{1}, r_{2}\right)$ by assumption, yielding the desired contradiction.

Since $s \geq u$, we immediately have

$$
\begin{aligned}
L_{1}[b, c] \geq \int_{\rho}^{1}\left(\left(b^{\prime}\right)^{2}+\left(c^{\prime}\right)^{2}+\frac{5}{r^{2}}\left(b^{2}\right.\right. & \left.+c^{2}\right)-\frac{8}{r^{2}} b c \\
& \left.+\lambda\left(6 u^{2}-1\right) b^{2}+\lambda\left(2 u^{2}-1\right) c^{2}\right) r \mathrm{~d} r \equiv m[b, c] .
\end{aligned}
$$

It remains to show that $\min \left\{m[b, c]: \int_{\Omega} b^{2}+c^{2} d A=1\right\}>0$, the proof of which follows by analogy with [34] with some technical differences, and we omit the details for brevity. The key steps are to compute the Euler-Lagrange equations associated with $b$ and $c$ in (31) and note that the system is satisfied weakly by the functions $b=u^{\prime}$ and $c=2 u / r$. We can thus manipulate the Euler-Lagrange equations for $b$ and $c$, exactly as in [34], to deduce the non-negativity of $L_{1}$.

The case $n=2$. The last step is to show that $L_{2}$ is positive for certain choices of $\lambda$ and $\rho$. First we note that

$$
L_{2}[b, c]=\int_{\rho}^{1}\left(\left(b^{\prime}\right)^{2}+\left(c^{\prime}\right)^{2}+\frac{8(b-c)^{2}}{r^{2}}+\lambda\left(6 s^{2}-1\right) b^{2}+\lambda\left(2 s^{2}-1\right) c^{2}\right) r \mathrm{~d} r .
$$

Now letting $b(r)=s(r) \alpha(r)$ and $c(r)=s(r) \beta(r)$ in (32) we can re-write $L_{2}$ as

$$
\begin{aligned}
L_{2}[s \alpha, s \beta] & =\int_{\rho}^{1} s^{2}\left[\left(\alpha^{\prime}\right)^{2}+\left(\beta^{\prime}\right)^{2}+\frac{4(2 \alpha-\beta)^{2}}{r^{2}}+\frac{4 \alpha^{2}}{r^{2}}\left(\lambda r^{2} s^{2}-3\right)\right] r \mathrm{~d} r \\
& \geq \int_{\rho}^{1} \frac{4 \alpha^{2} s^{2}}{r}\left(\lambda r^{2} s^{2}-3\right) \mathrm{d} r .
\end{aligned}
$$

We now recall that $s(r)$ satisfies the inequality (16), and it follows that $L_{2}>0$ provided $\lambda$ and $\rho$ satisfy the inequality (15a).

For the second inequality (15b), we use the fact that

$$
\int_{\rho}^{1}\left(u^{\prime}\right)^{2} r \mathrm{~d} r \geq \frac{\pi^{2}}{(\log \rho)^{2}} \int_{\rho}^{1} \frac{u^{2}}{r} \mathrm{~d} r
$$




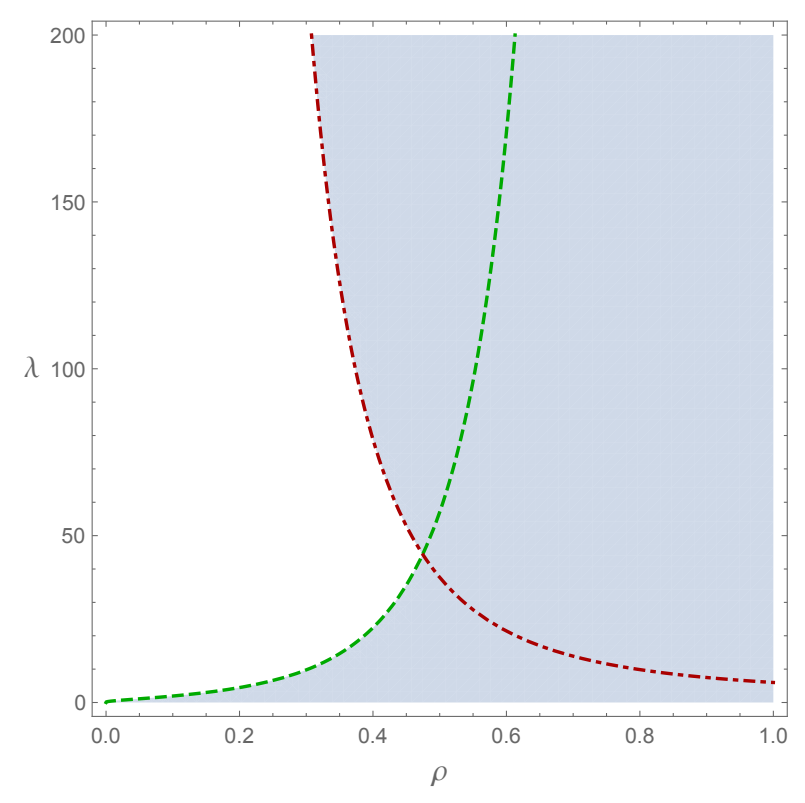

Figure 2: The $(\rho, \lambda)$ parameter space. The defect-free state is stable in the shaded region. The boundaries associated with the inequalities (15a) and (15b) are plotted as red, dot-dashed and green, dashed curves, respectively.

whenever $u(\rho)=u(1)=0$. Therefore from (32) we have

$$
L_{2}[b, c] \geq \int_{\rho}^{1}\left(\frac{\pi^{2}}{(\log \rho)^{2}}\left(b^{2}+c^{2}\right)+8(b-c)^{2}-\lambda r^{2}\left(1-2 s^{2}\right)\left(b^{2}+c^{2}\right)\right) \frac{\mathrm{d} r}{r},
$$

and it is easily shown that the integrand is positive definite provided

$$
\lambda<\frac{\pi^{2}}{r^{2}(\log \rho)^{2}\left(1-2 s^{2}\right)} .
$$

A lower bound on the right-hand side is found by setting $r=1$ and using the minimum value (16) for $s$, and it follows that (36) is satisfied uniformly whenever $\lambda$ satisfies the inequality (15b).

The proof of Proposition 1 is now complete.

Figure 2 shows the region of the $(\rho, \lambda)$ parameter space described by the two inequalities (15). Proposition 1 tells us that the defect-free state is stable in the shaded region. In particular, this shows that the defect-free state is stable whenever $\rho$ is sufficiently large, regardless of the size of $\lambda$. For smaller values of $\rho$, the defect-free state is stable both for sufficiently small and for sufficiently large values of $\lambda$. These observations can be explained using heuristic arguments. For example, one can prove that the $2 \mathrm{D}$ LdG energy has a unique critical point for either $\lambda$ sufficiently small or $\rho$ sufficiently close to 1 , following the arguments in [26] and [12]. For $\lambda$ large enough, we are deep in the nematic phase and defects are energetically expensive, so that the defect-free state is naturally preferred and hence, at least locally stable. These results will be further explored below in Sections 3.3 and 3.4 using numerical computations and formal asymptotic analysis. 


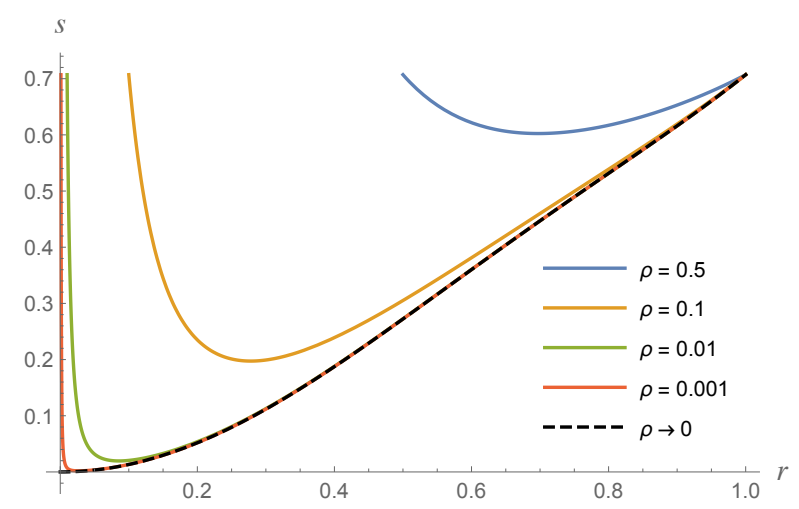

Figure 3: Defect-free state order parameter $s(r)$ versus $r$ for $\lambda=10$ and several values of $\rho$; the limiting solution as $\rho \rightarrow 0$ is shown as the black dashed curve.

\subsection{Numerical stability analysis}

Here we supplement Proposition 1 with a direct numerical investigation of the stability of the defect-free state. The first step is to compute the base state, namely the function $s(r)$ satisfying the boundary-value problem (12) and (14). We show some typical numerical solutions in Figure 3, with $\lambda=10$ and several different values of the inner radius $\rho$. When $\rho$ is close to 1 , the order parameter $s$ departs only slightly from its equilibrium value $1 / \sqrt{2}$, and the minimum value of $s$ decreases as $\rho$ decreases. As $\rho \rightarrow 0$, we see that $s(r)$ approaches a limiting solution corresponding to a disc in which $s(r) \rightarrow 0$ as $r \rightarrow 0$, and this limit will be analysed further below in Section 3.4.

Next, to determine the stability of the defect-free state, we examine when the second variation of the LdG energy ceases to be positive definite. We have demonstrated in Section 3.2 that it suffices to consider the functional $L_{2}[b, c]$ given by equation (32). The local stability of the defect-free state changes at critical values of $\rho$ and $\lambda$ for which the homogeneous Euler-Lagrange boundary-value problem corresponding to the functional $L_{2}[b, c]$, namely

$$
\begin{gathered}
b^{\prime \prime}+\frac{b^{\prime}}{r}+\frac{8}{r^{2}}(c-b)=\lambda\left(6 s^{2}-1\right) b, \\
c^{\prime \prime}+\frac{c^{\prime}}{r}+\frac{8}{r^{2}}(b-c)=\lambda\left(2 s^{2}-1\right) c, \\
b(\rho)=c(\rho)=b(1)=c(1)=0,
\end{gathered}
$$

admits nontrivial solutions.

We solve (37) as an eigenvalue problem for $\lambda$ and $\rho$. Given $\lambda$, a basis for the solution space is $\left\{b_{1}(r), c_{1}(r)\right\}$ and $\left\{b_{2}(r), c_{2}(r)\right\}$, where the functions $b_{k}, c_{k}$ satisfy the differential equations $(37 \mathrm{a}, \mathrm{b})$ and the boundary conditions

$$
\begin{array}{llll}
b_{1}(1)=0, & b_{1}^{\prime}(1)=1, & c_{1}(1)=0, & c_{1}^{\prime}(1)=0, \\
b_{2}(1)=0, & b_{2}^{\prime}(1)=0, & c_{2}(1)=0, & c_{2}^{\prime}(1)=1 .
\end{array}
$$

These basis functions automatically satisfy the boundary conditions at $r=1$, and a linear combination of these functions also satisfies the homogeneous boundary conditions at $r=\rho$ if and only if

$$
\Delta(\rho)=b_{1}(\rho) c_{2}(\rho)-b_{2}(\rho) c_{1}(\rho)=0
$$




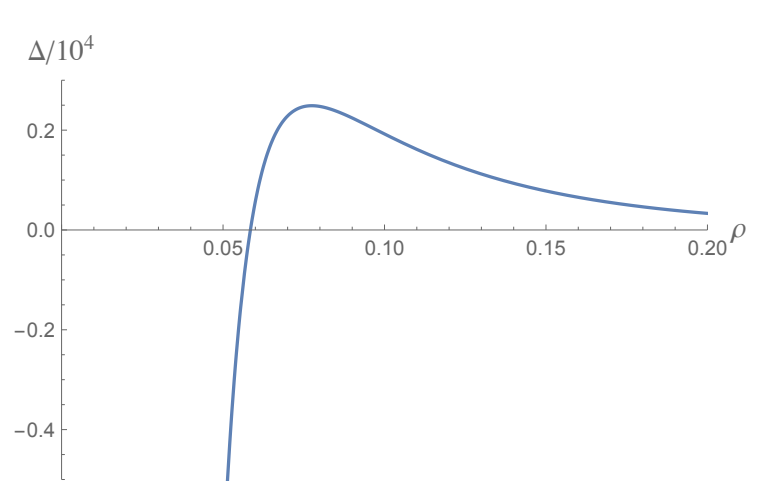

(a) $\lambda=30$ : here $\Delta=0$ at $\rho \approx 0.5847$.

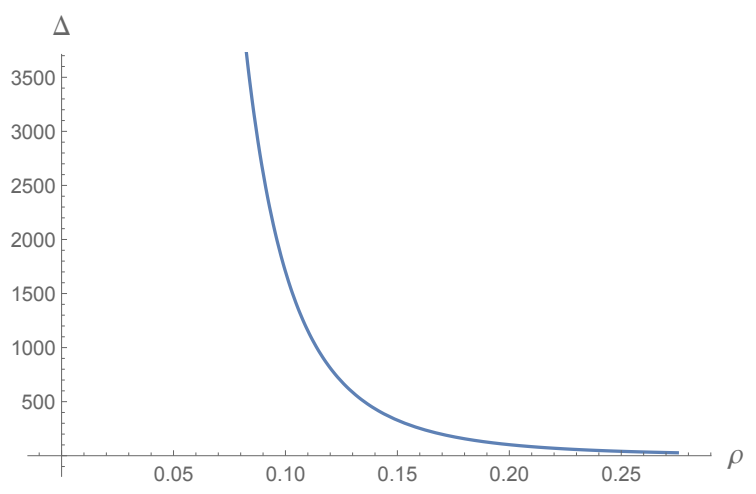

(b) $\lambda=5$ : here $\Delta$ is positive for all $\rho$.

Figure 4: Discriminant $\Delta$ versus inner radius $\rho$ for (a) $\lambda=30$ and (b) $\lambda=5$.

Figure 4(a) shows a graph of the function $\Delta(\rho)$ with $\lambda=30$, and we see that $\Delta(\rho)$ crosses zero at $\rho \approx 0.5847$. In contrast, Figure 4 (b) shows the corresponding behaviour with $\lambda=5$, indicating that in this case $\Delta$ remains positive for all $\rho$.

By computing for each value of $\lambda$ the value of $\rho$ where $\Delta=0$ (if it exists), we generate a curve in the $(\rho, \lambda)$ plane, as shown in Figure 5 . This curve separates the regions of parameter space in which the defect-free state is locally stable and unstable. The analysis of Section 3.2 shows that the defect-free state is stable as $\lambda \rightarrow \infty$ for fixed $\rho$, and we infer that the stable region is to the right of the curve in Figure 5 and unstable to the left. We note that the curve in Figure 5 appears to terminate with $\rho \rightarrow 0$ at a finite value of $\lambda \approx 7.43472$, in agreement with the observation in Figure 4(b) that $\Delta$ appears never to change sign when $\lambda=5$. We also observe that the critical value of $\rho$ seems to approach zero as $\lambda \rightarrow \infty$. Both of these observations may be corroborated by asymptotic analysis of the problem in the limit $\rho \rightarrow 0$, as shown below in Section 3.4.

To provide further evidence that the local stability is as depicted in Figure 5, we now analyse how the sign of the functional $L_{2}$ varies in a neighbourhood of the marginal curve. Let us denote the critical value of $\lambda$ for a given value of $\rho$ by the two-branched function $\lambda=\lambda^{*}(\rho)$. At this critical value of $\lambda$, the boundary-value problem (37) admits nontrivial solutions, which we denote by $\{b, c\}=\left\{b^{*}(r), c^{*}(r)\right\}$. Likewise we denote by $s=s^{*}$ the solution of the boundary-value problem (12),(14) with $\lambda=\lambda^{*}$ (naturally, these solutions also vary parametrically with $\rho$ ).

When evaluated at the function pair $\left\{b^{*}(r), c^{*}(r)\right\}$, the functional $L_{2}$ (referring to (32) and (37)) takes the form

$$
L_{2}\left[b^{*}, c^{*}\right]=\int_{\rho}^{1}\left(b^{* 2}\left[\lambda\left(6 s^{2}-1\right)-\lambda^{*}\left(6 s^{* 2}-1\right)\right]+c^{* 2}\left[\lambda\left(2 s^{2}-1\right)-\lambda^{*}\left(2 s^{* 2}-1\right)\right]\right) r \mathrm{~d} r .
$$

We examine the local behaviour of this function close to the critical value $\lambda=\lambda^{*}$ by setting

$$
\lambda-\lambda^{*}=\delta, \quad s-s^{*}=\delta \tilde{s},
$$

and linearising with respect to the small perturbation $\delta$. We find that the right-hand side of (40) reduces to

$$
L_{2}\left[b^{*}, c^{*}\right] \sim \delta F_{2}(\rho)
$$




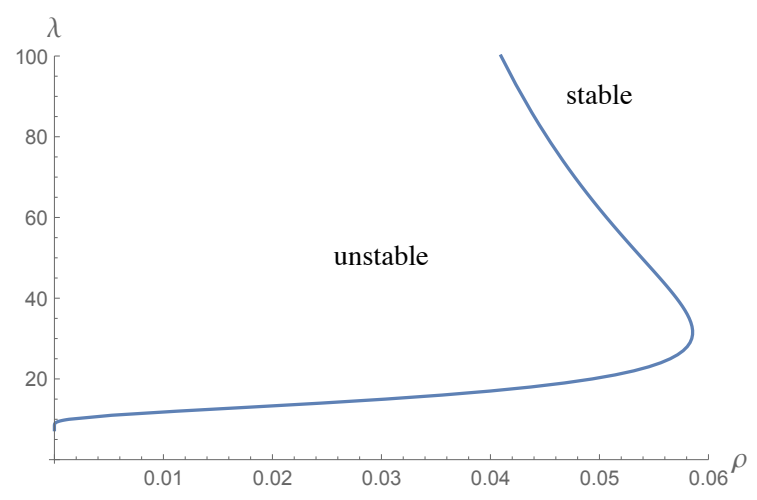

(a)

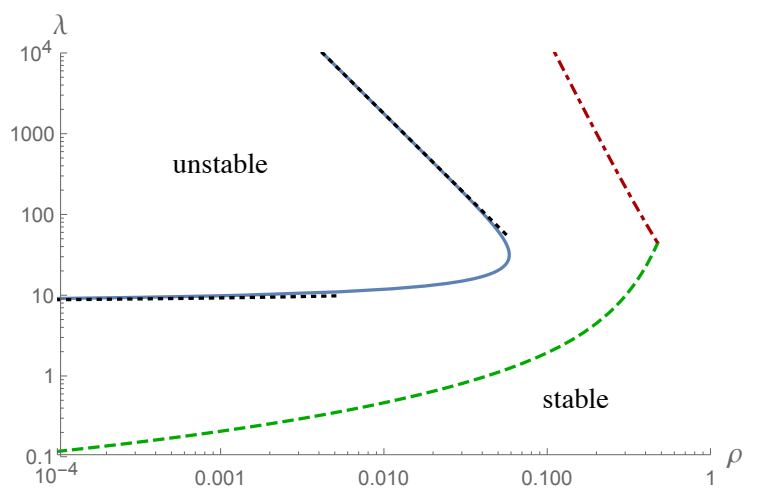

(b)

Figure 5: (a) The $(\rho, \lambda)$ parameter space, showing the marginal stability curve where the discriminant $\Delta$ is equal to zero. (b) The same parameter space plotted with logarithmic axes; the limiting behaviours (55) and (67) as $\rho \rightarrow 0$ are plotted as black dotted curves; the analytical stability boundaries corresponding to (15) are shown as green dashed and red dot-dashed curves. The logarithmic axes in Figure 5(b) show that the upper branch of the stability curve has $\rho$ proportional to a negative power of $\lambda$ as $\lambda \rightarrow \infty$.

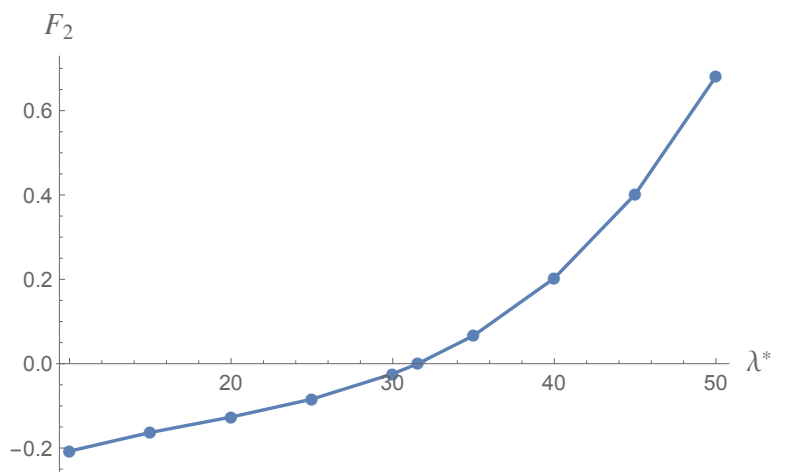

Figure 6: The functional $F_{2}(\rho)$ defined by equation (43) plotted versus $\lambda^{*}(\rho)$.

where

$$
F_{2}(\rho)=\int_{\rho}^{1}\left(b^{* 2}\left[\left(6 s^{* 2}-1\right)+12 \lambda^{*} s^{*} \tilde{s}\right]+c^{* 2}\left[\left(2 s^{* 2}-1\right)+4 \lambda^{*} s^{*} \tilde{s}\right]\right) r \mathrm{~d} r
$$

and $\tilde{s}(r)$ satisfies the homogeneous boundary-value problem

$$
\begin{gathered}
\tilde{s}^{\prime \prime}+\frac{\tilde{s}^{\prime}}{r}-\frac{4 \tilde{s}}{r^{2}}-\lambda^{*}\left(6 s^{* 2}-1\right) \tilde{s}=s^{*}\left(2 s^{* 2}-1\right), \\
\tilde{s}(\rho)=\tilde{s}(1)=0 .
\end{gathered}
$$

The variation in stability as the critical curve is crossed depends on the sign of $F_{2}$. If $F_{2}$ is negative, then $L_{2}$ ceases to be positive definite, and the defect-free state loses stability, as $\lambda$ increases through the critical value $\lambda^{*}$. Conversely, if $F_{2}$ is positive, then the defect-free state loses stability as $\lambda$ decreases through $\lambda^{*}$.

For a given value of $\rho$, we numerically solve for $\lambda^{*}, s^{*}, b^{*}$ and $c^{*}$ as described above, choosing to normalise $b^{*}$ and $c^{*}$ such that $b^{* \prime}(1)=1$. We simultaneously solve (44) for $\tilde{s}$ and 
substitute into (43) to evaluate $F_{2}(\rho)$. We then plot $F_{2}(\rho)$ versus $\lambda^{*}(\rho)$ parametrically in Figure 6 . We observe that $F_{2}<0$ when $\lambda^{*}<\lambda_{\mathrm{c}}$ and $F_{2}>0$ when $\lambda^{*}>\lambda_{\mathrm{c}}$, where $\lambda_{\mathrm{c}} \approx 31.55$ is precisely the value of $\lambda$ at the "nose" of the critical stability curve plotted in Figure 5 . We deduce that the defect-free state loses stability as $\lambda$ increases through the lower branch of the marginal curve (where $\lambda^{*}<\lambda_{\mathrm{c}}$ ), or as $\lambda$ decreases though the upper branch of the marginal curve, in agreement with the labels in Figure 5(a).

\subsection{Asymptotic analysis}

To explain and validate the behaviour observed in Figure 5, we now examine the asymptotic limit where $\rho \rightarrow 0$. In this limit, the central hole shrinks to a point and the annulus approaches a disc. The presence of the hole remains noticeable in an inner region which is analysed using the scalings

$$
r=\rho x, \quad s(r)=S(x) .
$$

The Euler-Lagrange equation (14) is transformed to

$$
S^{\prime \prime}+\frac{S^{\prime}}{x}-\frac{4 S}{x^{2}}+\lambda \rho^{2}\left(1-2 S^{2}\right) S=0,
$$

subject to $S(1)=1 / \sqrt{2}$ and suitable matching conditions as $x \rightarrow \infty$.

It is straightforward to solve equation (46) in the limit $\rho \rightarrow 0$ as an asymptotic expansion in powers of $\rho^{2}$, namely

$$
S(x) \sim \frac{1}{\sqrt{2} x^{2}}+\rho^{2}\left[\frac{\lambda}{12 \sqrt{2}}\left(3-\frac{4}{x^{2}}+\frac{1}{x^{4}}\right)+K\left(x^{2}-\frac{1}{x^{2}}\right)\right]+O\left(\rho^{4}\right),
$$

where $K$ is an arbitrary integration constant. We note that the expansion (47) is nonuniform when $\lambda=O\left(\rho^{-2}\right)$, and this represents an alternative distinguished limit to be analysed separately below.

Now returning to the outer region where $r=O(1)$ and matching with the inner solution (47), we obtain modified effective boundary conditions for the Euler-Lagrange equation (14), namely

$$
s(r) \sim K r^{2} \quad \text { as } r \rightarrow 0, \quad s(1)=\frac{1}{\sqrt{2}} .
$$

This modified boundary-value problem may be solved numerically for any given value of $\lambda$, with the corresponding value of the coefficient $K$ determined as part of the solution. The resulting limiting outer solution for $\lambda=10$ is shown as the black dashed curve in Figure 3.

Now we express the perturbed problem (37) in inner variables:

$$
\begin{aligned}
& B^{\prime \prime}+\frac{B^{\prime}}{x}+\frac{8}{x^{2}}(C-B)=\lambda \rho^{2}\left(6 S^{2}-1\right) B, \\
& C^{\prime \prime}+\frac{C^{\prime}}{x}+\frac{8}{x^{2}}(B-C)=\lambda \rho^{2}\left(2 S^{2}-1\right) C,
\end{aligned}
$$

where $B(x)=b(r)$ and $C(x)=c(r)$. Letting $\rho \rightarrow 0$, we find the leading-order inner solution satisfying the boundary conditions $B(1)=C(1)=0$ takes the form

$$
B(x) \sim M_{1} \log x+M_{2}\left(x^{4}-\frac{1}{x^{4}}\right), \quad C(x) \sim M_{1} \log x-M_{2}\left(x^{4}-\frac{1}{x^{4}}\right),
$$




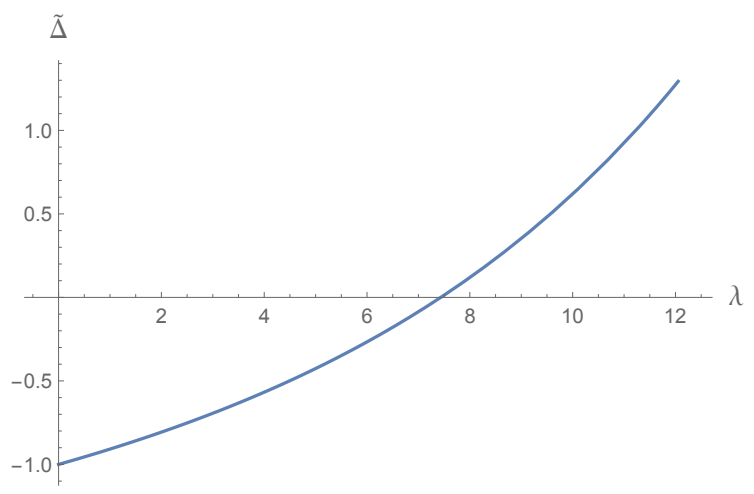

(a)

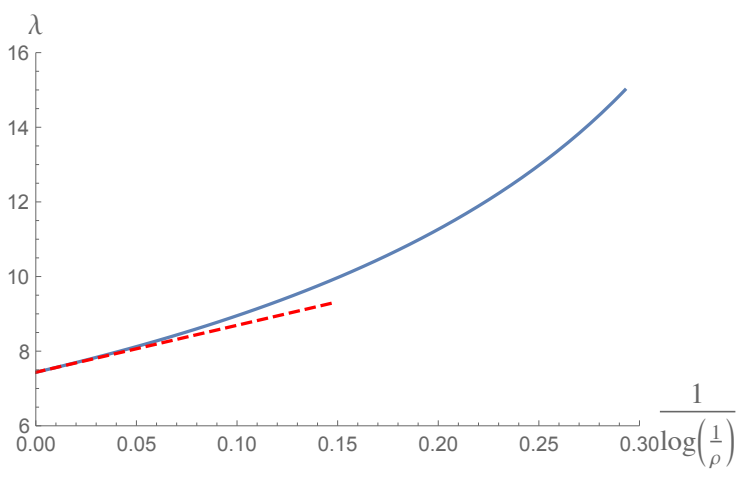

(b)

Figure 7: (a) Discriminant $\tilde{\Delta}$ versus $\lambda$; we find that $\tilde{\Delta}=0$ at $\lambda \approx 7.43472$. (b) Relation between $\lambda$ and $\rho$ implied by equation (53); the fit (55) is shown as the red dashed curve.

where $M_{1}, M_{2}$ are arbitrary constants.

Reverting to the outer variables, we deduce that the leading-order outer solution $\{b(r), c(r)\}$ may be written in the form

$$
\left(\begin{array}{l}
b(r) \\
c(r)
\end{array}\right)=m_{1}\left(\begin{array}{l}
\mathrm{b}_{1}(r) \\
\mathrm{c}_{1}(r)
\end{array}\right)+m_{1} \log (1 / \rho)\left(\begin{array}{l}
\mathrm{b}_{2}(r) \\
\mathrm{c}_{2}(r)
\end{array}\right)+m_{2}\left(\begin{array}{l}
\mathrm{b}_{3}(r) \\
\mathrm{c}_{3}(r)
\end{array}\right),
$$

where $m_{1}, m_{2}$ are constants. The functions $\left\{\mathbf{b}_{j}, \mathrm{c}_{j}\right\}$ are three canonical solutions of the outer equations $(37 \mathrm{a}, \mathrm{b})$ subject to the matching conditions

$$
\begin{array}{ll}
\left(\begin{array}{l}
\mathrm{b}_{1}(r) \\
\mathrm{c}_{1}(r)
\end{array}\right) \sim\left(\begin{array}{l}
1 \\
1
\end{array}\right)\left[\log r+\frac{\lambda r^{2}}{4}(1-\log r)+\cdots\right] & \text { as } r \rightarrow 0, \\
\left(\begin{array}{l}
\mathrm{b}_{2}(r) \\
\mathrm{c}_{2}(r)
\end{array}\right) \sim\left(\begin{array}{c}
1 \\
1
\end{array}\right)\left[1-\frac{\lambda r^{2}}{4}+\cdots\right] & \text { as } r \rightarrow 0, \\
\left(\begin{array}{c}
\mathrm{b}_{3}(r) \\
\mathrm{c}_{3}(r)
\end{array}\right) \sim\left(\begin{array}{c}
1 \\
-1
\end{array}\right)\left[r^{4}-\frac{\lambda r^{6}}{20}+\cdots\right] & \text { as } r \rightarrow 0 .
\end{array}
$$

These canonical functions may be computed numerically for any given value of $\lambda$. The boundary conditions $b(1)=c(1)=0$ then imply the solvability condition

$$
\log (1 / \rho)\left[b_{2}(1) c_{3}(1)-b_{3}(1) c_{2}(1)\right]+b_{1}(1) c_{3}(1)-b_{3}(1) c_{1}(1)=0,
$$

which is accurate up to algebraic corrections in $\rho$.

In the limit as $\rho \rightarrow 0$, the solvability condition (53) reduces to

$$
\tilde{\Delta}(\lambda)=\mathrm{b}_{2}(1) \mathrm{c}_{3}(1)-\mathrm{b}_{3}(1) \mathrm{c}_{2}(1)=0 .
$$

We plot this modified discriminant $\tilde{\Delta}$ versus $\lambda$ in Figure $7($ a). We find that $\tilde{\Delta}$ crosses zero at $\lambda \approx 7.43472$, in agreement with minimum value of $\lambda$ on the marginal stability curve in Figure 5 .

When $\rho$ is small but nonzero, we can extract the limiting relation between $\lambda$ and $\rho$ using the full solvability condition (53). The resulting behaviour is plotted in Figure 7(b), along with the fit

$$
\lambda \sim 7.43472+\frac{12.58825}{\log (1 / \rho)} \quad \text { as } \rho \rightarrow 0 .
$$


This asymptotic solution is shown as a black dashed curve in Figure 5(b), and we see that it successfully captures the local behaviour as $\rho \rightarrow 0$, as well as confirming that the marginal stability curve indeed terminates at the finite value $\lambda \approx 7.43472$.

As noted above, an alternative distinguished limit occurs when $\lambda=O\left(1 / \rho^{2}\right)$ as $\rho \rightarrow 0$. The defect-free state is found by solving the Euler-Lagrange equation (14). With $\lambda \gg 1$, the solution for $s(r)$ is easily obtained as an asymptotic expansion in inverse powers of $\lambda$, namely

$$
s(r) \sim \frac{1}{\sqrt{2}}-\frac{\sqrt{2}}{\lambda r^{2}}+O\left(\lambda^{-2}\right) .
$$

The failure of this outer solution to satisfy the boundary condition $s(1)=1 / \sqrt{2}$ is easily resolved by considering a boundary layer at $r=1$.

We focus instead on the inner region close to $r=\rho$ by performing the scaling

$$
r=\frac{y}{\sqrt{\lambda}}, \quad s(r)=S(y),
$$

so that equation (14) is transformed to

$$
S^{\prime \prime}+\frac{S^{\prime}}{y}-\frac{4 S}{y^{2}}-\left(1-2 S^{2}\right) S=0 .
$$

Matching with the outer solution (56) implies the far-field behaviour

$$
S(y) \sim \frac{1}{\sqrt{2}}-\frac{\sqrt{2}}{y^{2}}-\frac{3 \sqrt{2}}{y^{4}}+\cdots+\frac{\tilde{K}}{\sqrt{y}} \mathrm{e}^{-y \sqrt{2}} \text { as } y \rightarrow \infty,
$$

where $\tilde{K}$ is an arbitrary shooting parameter. The solution is found numerically by integrating from large positive $y$ towards $y=0$. The integration is halted when $S=1 / \sqrt{2}$ and we read off

$$
S(\mu)=\frac{1}{\sqrt{2}} \quad \text { where } \quad \mu=\rho \sqrt{\lambda}=O(1) .
$$

By varying the shooting parameter $\tilde{K}$, we generate the inner solutions corresponding to different values of $\mu$.

Having solved for the defect-free order parameter $s(r)$, we next turn our attention to the perturbation functions $b(r)$ and $c(r)$. Equations $(37 \mathrm{a}, \mathrm{b})$ are easily solved in the limit $\lambda \rightarrow \infty$ to get the outer solutions

$$
b(r) \sim \frac{4}{\lambda r^{2}}\left(\frac{1}{r^{2}}-r^{2}\right), \quad c(r) \sim \frac{1}{r^{2}}-r^{2} .
$$

In the inner region, we perform the rescalings

$$
r=\frac{y}{\sqrt{\lambda}}, \quad b(r)=\lambda B(y), \quad c(r)=\lambda C(y)
$$

to get the inner equations

$$
\begin{aligned}
& B^{\prime \prime}+\frac{B^{\prime}}{y}+\frac{8}{y^{2}}(C-B)=\left(6 S^{2}-1\right) B \\
& C^{\prime \prime}+\frac{C^{\prime}}{y}+\frac{8}{y^{2}}(B-C)=\left(2 S^{2}-1\right) C .
\end{aligned}
$$




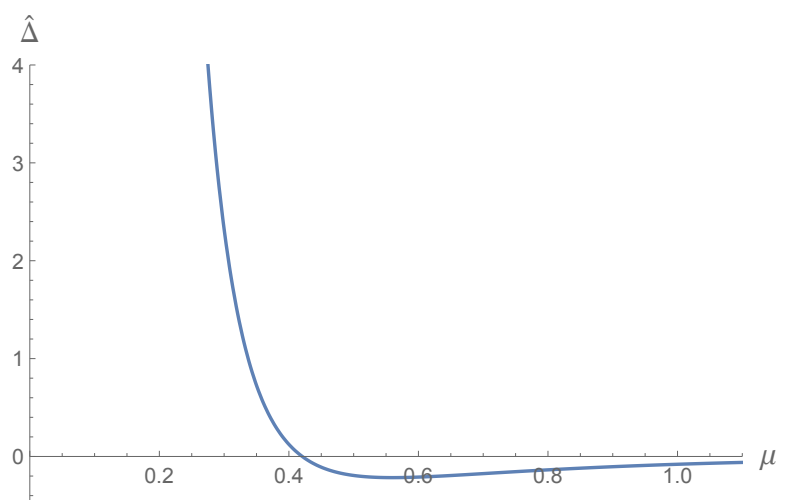

Figure 8: Modified discriminant $\hat{\Delta}$ versus scaled inner radius $\mu=\rho \sqrt{\lambda}$, showing that $\hat{\Delta}$ crosses zero at $\mu \approx 0.42065$.

Matching with the outer solution (61) imposes the far-field conditions $B(y) \sim 4 / y^{4}$ and $C(y) \sim 1 / y^{2}$ as $y \rightarrow \infty$. It is helpful to decompose the inner solutions into two components with algebraic and exponential far-field behaviour. To this end, we write

$$
B(y)=B_{a}(y)+B_{e}(y), \quad C(y)=C_{a}(y)+C_{e}(y),
$$

where $\left\{B_{a}(y), C_{a}(y)\right\}$ and $\left\{B_{e}(y), C_{e}(y)\right\}$ are solutions of the differential equations (63) subject to the far-field conditions

$$
\begin{aligned}
B_{a}(y) & \sim \frac{4}{y^{4}}+\frac{80}{3 y^{6}}+\cdots, & B_{e}(y) & \sim \frac{\mathrm{e}^{-y \sqrt{2}}}{\sqrt{y}}, \\
C_{a}(y) & \sim \frac{1}{y^{2}}-\frac{10}{3 y^{4}}+\cdots, & C_{e}(y) & \sim-\frac{4 \mathrm{e}^{-y \sqrt{2}}}{y^{5 / 2}}
\end{aligned}
$$

as $y \rightarrow \infty$. We solve numerically for $\left\{B_{a}(y), C_{a}(y), B_{e}(y), C_{e}(y)\right\}$, and the boundary conditions $B(\mu)=C(\mu)=0$ can then be satisfied if and only if

$$
\hat{\Delta}=B_{a}(\mu) C_{e}(\mu)-B_{e}(\mu) C_{a}(\mu)=0 .
$$

We plot $\hat{\Delta}$ versus $\mu$ in Figure 8 . We find that $\hat{\Delta}=0$ at a critical value $\rho \sqrt{\lambda}=\mu \approx 0.42065$, and we infer that the asymptotic behaviour of the marginal curve as $\rho \rightarrow 0$ and $\lambda \rightarrow \infty$ is approximated by

$$
\lambda \sim \frac{0.177}{\rho^{2}} \text { as } \rho \rightarrow 0
$$

The excellent agreement between this predicted behaviour and our numerically computed marginal curve is indicated by a black dashed curve in Figure 5(b).

\subsection{Discussion}

In Section 3.3, we compute a sharp stability boundary for the defect-free state in terms of the inner radius $\rho$ and the material parameter $\lambda$. The resulting marginal curve shown in Figure 5 has several interesting features. 
1. There is a maximum value of $\rho \approx 0.05855$ on the marginal curve: if the inner radius exceeds this critical value, then the defect-free state is stable regardless of the value of $\lambda$.

2. As $\lambda$ decreases, the marginal curve terminates with $\rho \searrow 0$ as $\lambda \searrow 7.43472$. This was confirmed in Section 3.4 using asymptotic analysis, which correctly predicted the local behaviour (55). The implication is that the defect-free state is stable for all $\rho$ if $\lambda<7.43472$.

3. When $\lambda \gg 1$, the marginal value of $\rho$ decreases towards zero. Again, we confirmed this observation using asymptotic analysis and found the limiting behaviour (67).

In Section 3.2, we prove that the defect-free state is locally stable if the inequalities (15) are satisfied, which corresponds to the region to the right of the green dashed and red dotdashed curves in Figure 5(b). We see in Figure 5(b) that the rigorously proven region of stabilty is indeed a subset of the numerically computed region of stability. The quantified asymptotic behaviour (67) of the marginal curve establishes that the red and blue curves shown in Figure 5(b) cannot intersect as $\lambda \rightarrow \infty$.

Finally, we further validate the predictions of this section by performing simulations of the gradient-flow problem for the full 2D LdG problem, with a defect-free initial condition, in an annulus with $\rho=0.05$ and different values of $\lambda$. The results are shown in Figure 9. With this value of $\rho$, the stability curve plotted in Figure 5 tells us that the defect-free state should be stable for $\lambda \lesssim 20.29$ and $\lambda \gtrsim 62.12$, and unstable for $20.29 \lesssim \lambda \lesssim 62.12$.

Considering first the top row of Figure 9 , we see that the equilibrium is radially symmetric and defect-free when $\lambda=20$, as expected. As $\lambda$ increases through the stability boundary, we observe the loss of radial symmetry, which culminates in the formation of two internal defects. The second row of Figure 9 shows that the defects remain in roughly the same locations as $\lambda$ increases further, while becoming noticeably stronger (in terms of reduced order) and more localized. Finally, the bottom row of Figure 9 shows that the equilibrium transitions rapidly back to the defect-free state as $\lambda$ increases through the upper branch of the stability boundary, and the cost in energy associated with the increasingly strong defects ceases to be worth paying.

\section{The defect-free state in the 2D LdG framework and radially symmetric solutions in the 3D LdG framework}

In this section, we discuss the relevance of the results in Section 3 to the 3D LdG framework. Our aim is to relate the defect-free state $\mathbf{Q}^{*}$ defined in (11) to an exact solution of the LdG Euler-Lagrange equations in (4). With $\mathbf{Q}$ again normalized with $\sqrt{|A| / C}$, the 3D LdG energy (1) takes the form

$$
I[\mathbf{Q}]=\int_{\Omega} \frac{1}{2}|\nabla \mathbf{Q}|^{2}+\lambda\left[-\frac{1}{2} \operatorname{tr} \mathbf{Q}^{2}-\frac{\beta}{3} \operatorname{tr} \mathbf{Q}^{3}+\frac{1}{4}\left(\operatorname{tr} \mathbf{Q}^{2}\right)^{2}\right] \mathrm{d} \Omega,
$$

where $\lambda=|A| R_{\text {out }}^{2} / L$, as above, and $\beta=B / \sqrt{C|A|}$. The corresponding normalized EulerLagrange equations (4) read

$$
\Delta \mathbf{Q}_{i j}=\lambda\left[\mathbf{Q}_{i j}-\beta\left(\mathbf{Q}_{i p} \mathbf{Q}_{p j}-\operatorname{tr} \mathbf{Q}^{2} \frac{\delta_{i j}}{3}\right)+|\mathbf{Q}|^{2} \mathbf{Q}_{i j}\right] .
$$




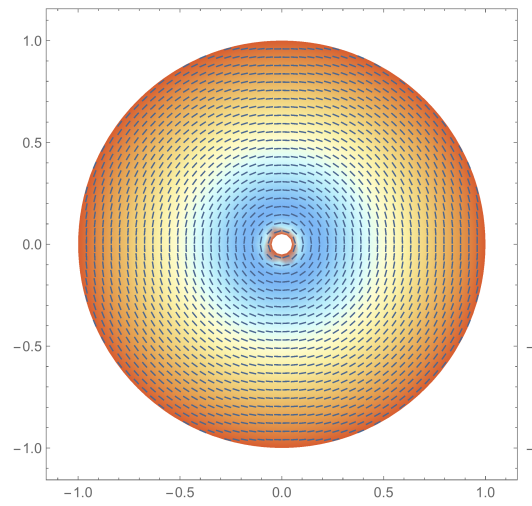

(a) $\lambda=20$

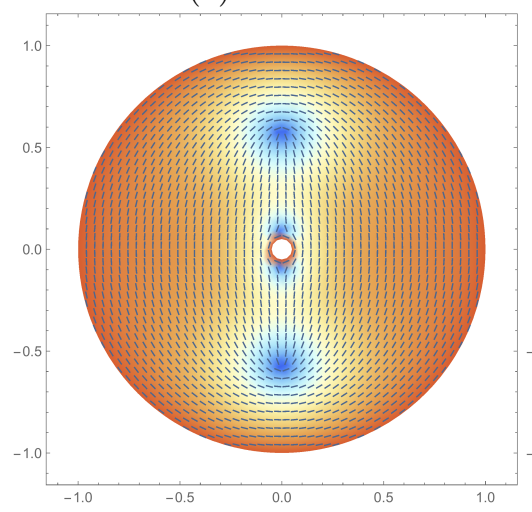

(d) $\lambda=30$

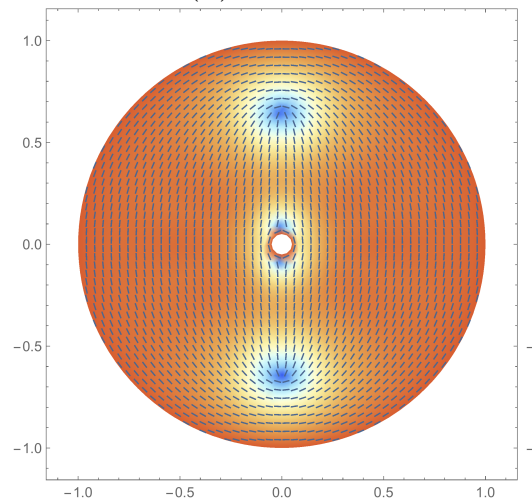

(g) $\lambda=60$

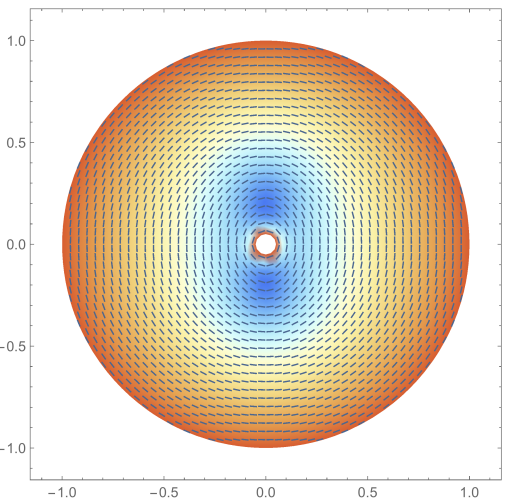

(b) $\lambda=20.5$

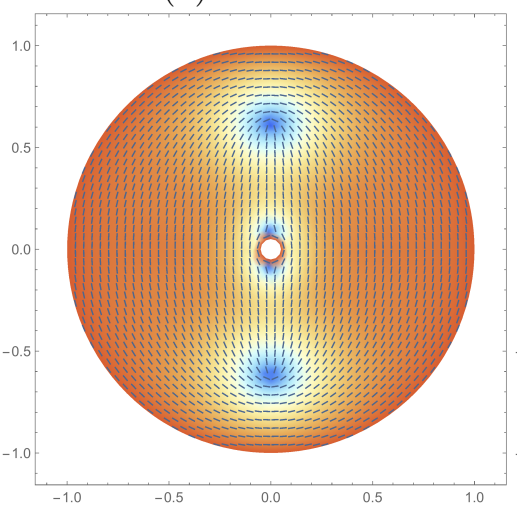

(e) $\lambda=40$

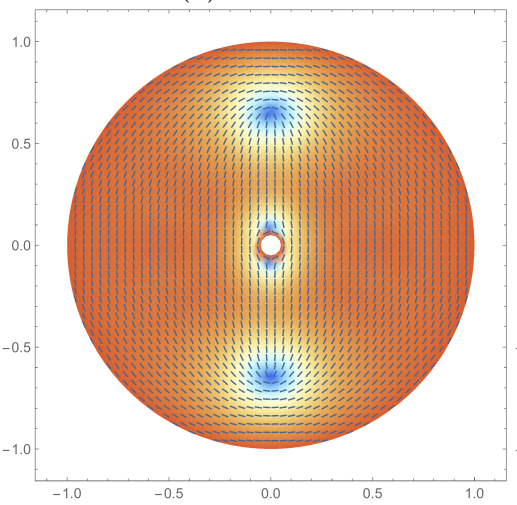

(h) $\lambda=62$

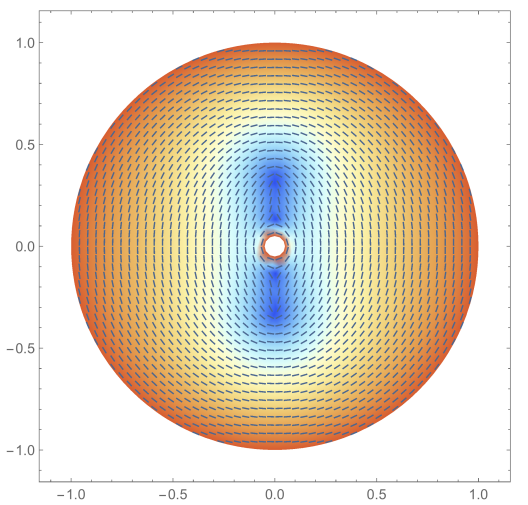

(c) $\lambda=21$

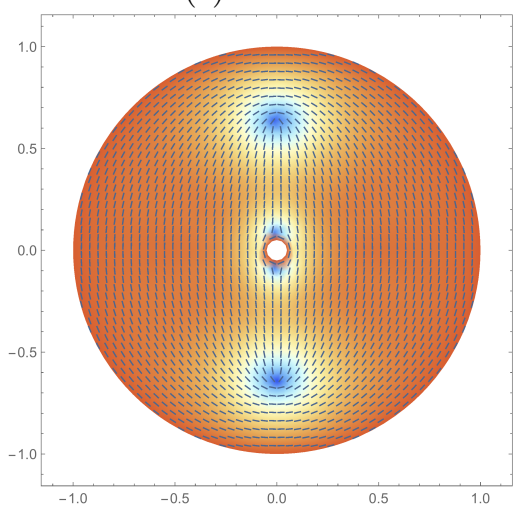

(f) $\lambda=50$

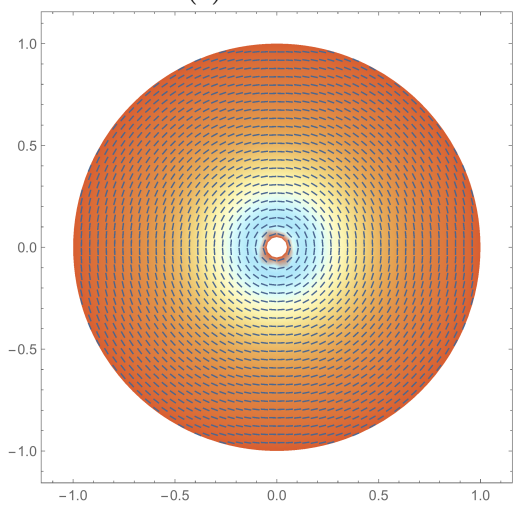

(i) $\lambda=63$

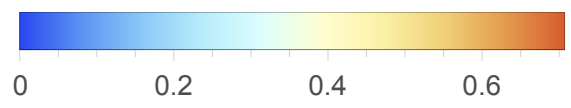

Colour scale for order parameter $s$.

Figure 9: Simulations of the gradient-flow model for the 2D LdG energy with $\rho=0.05$ and different values of $\lambda$. 
We define the orthonormal triad

$$
\mathbf{n}=(\cos \phi, \sin \phi, 0), \quad \mathbf{m}=(-\sin \phi, \cos \phi, 0), \quad \mathbf{p}=(0,0,1),
$$

where as before $\phi$ is the usual cylindrical polar angle (note that $\mathbf{n}$ and $\mathbf{m}$ are analogous to the $2 \mathrm{D}$ vectors $\mathbf{e}_{r}$ and $\mathbf{e}$ introduced in Sections 2 and 3$)$. A basis for the set of symmetric, traceless $3 \times 3$ matrices is given by

$$
\begin{array}{cc}
\mathbf{E}_{1}=(\mathbf{n} \otimes \mathbf{n}-\mathbf{m} \otimes \mathbf{m}) ; & \mathbf{E}_{2}=(\mathbf{n} \otimes \mathbf{m}+\mathbf{m} \otimes \mathbf{n}) ; \\
\mathbf{E}_{3}=(\mathbf{n} \otimes \mathbf{p}+\mathbf{p} \otimes \mathbf{n}) ; & \mathbf{E}_{4}=(\mathbf{m} \otimes \mathbf{p}+\mathbf{p} \otimes \mathbf{m}) \\
\mathbf{E}_{5}=\left(\mathbf{p} \otimes \mathbf{p}-\frac{\mathbf{I}_{3}}{3}\right),
\end{array}
$$

where $\mathbf{I}_{3}$ is the $3 \times 3$ identity matrix. Then any arbitrary symmetric, traceless $3 \times 3$ matrix can be written as

$$
\mathbf{Q}=\sum_{i=1}^{5} q_{i}(r, \phi, z) \mathbf{E}_{i} .
$$

In [16], the authors show that there exists a class of radially symmetric solutions of (69) labelled by two scalar order parameters, $u$ and $v$, of the form

$$
\mathbf{Q}_{u, v}=\frac{u(r)}{2} \mathbf{E}_{1}+v(r) \mathbf{E}_{5} .
$$

In [20], the authors prove that such solutions are unstable on $\mathbb{R}^{2}$ and in [22], the authors study the stability of these solutions in terms of an anisotropy parameter $M$, the disc radius $R$ and specific values of $\beta$ in (68). We do not make specific comparisons to the results in $[20,22]$ because $M=0$ in our case and our domain is a re-scaled annulus (not a disc) with a dimensionless geometrical parameter $\rho$. More generally, we note that solutions of the form $\mathbf{Q}=u(x, y) \mathbf{E}_{1}+w(x, y) \mathbf{E}_{2}+v(x, y) \mathbf{E}_{5}$ have been studied in [6] as minimizers of the Landau-de Gennes energy on 2D bounded simply-connected domains, $\Omega$, for topologically non-trivial Dirichlet conditions in the limit of vanishing elastic constant. We do not appeal to their results since the annulus is not simply connected in 2D and we are not working in the limit of vanishing elastic constant; our aim is to understand the relevance of $2 \mathrm{D}$ studies such as the one in Section 3 in a 3D setting.

With the $\mathbf{Q}$-tensor of the form $\mathbf{Q}_{u, v}$, the 3D LdG energy (68) is proportional to

$$
\begin{array}{r}
\mathcal{E}[u, v]=\int_{\rho}^{1}\left\{\frac{1}{4}\left(u^{\prime}\right)^{2}+\frac{1}{3}\left(v^{\prime}\right)^{2}+\frac{u^{2}}{r^{2}}+\lambda\left(-\frac{u^{2}}{4}-\frac{v^{2}}{3}+\frac{u^{4}}{16}+\frac{u^{2} v^{2}}{6}+\frac{v^{4}}{9}\right)\right. \\
\left.-\frac{\lambda \beta}{3} v\left(\frac{2 v^{2}}{9}-\frac{u^{2}}{2}\right)\right\} r \mathrm{~d} r .
\end{array}
$$

The scalar order parameters $u$ and $v$ are defined to be minimizers of $\mathcal{E}[u, v]$ in an appropriately defined admissible space and are, consequently, classical solutions of the following system of coupled ordinary differential equations:

$$
\begin{aligned}
u^{\prime \prime}+\frac{u^{\prime}}{r}-\frac{4 u}{r^{2}} & =\lambda u\left[-1+\frac{2 \beta}{3} v+\frac{u^{2}}{2}+\frac{2 v^{2}}{3}\right], \\
v^{\prime \prime}+\frac{v^{\prime}}{r} & =\lambda v\left[-1-\frac{\beta}{3} v+\frac{u^{2}}{2}+\frac{2 v^{2}}{3}\right]+\frac{\lambda \beta}{4} u^{2} .
\end{aligned}
$$


The 3D Dirichlet condition is $\mathbf{Q}=\mathbf{Q}_{b}$ on $r=\rho, 1$ where

$$
\mathbf{Q}_{b}=s_{+}\left(\mathbf{m} \otimes \mathbf{m}-\frac{\mathbf{I}_{3}}{3}\right) ; \quad s_{+}=\frac{1}{4}\left(\beta+\sqrt{\beta^{2}+24}\right)
$$

is a minimizer of the bulk potential. The corresponding boundary conditions for $u$ and $v$ are

$$
u(\rho)=u(1)=-s_{+} ; \quad v(\rho)=v(1)=-\frac{s_{+}}{2} .
$$

Let us work at the fixed temperature $A=-B^{2} /(3 C)$, corresponding to $\beta=\sqrt{3}$ and $s_{+}=\sqrt{3}$. Then it is straightforward to check that $v(r) \equiv-\sqrt{3} / 2$ identically satisfies both the Euler-Lagrange equation (75b) and the boundary conditions (77). Therefore

$$
\mathbf{Q}^{*}=\frac{u(r)}{2} \mathbf{E}_{1}-\frac{\sqrt{3}}{2} \mathbf{E}_{5}
$$

gives an exact solution of the 3D LdG Euler-Lagrange equations in (69) at this fixed temperature. The existence of exact solutions of the LdG Euler-Lagrange equations with constant components along some eigendirections at $A=-B^{2} /(3 C)$, was first noted in [11]. The scalar order parameter $u$ is interpreted to be a minimizer of the energy $\mathcal{E}(u, v)$ in $(74)$ with $v=-\sqrt{3}$, i.e.

$$
\mathcal{E}[u]:=\int_{\rho}^{1}\left(\frac{1}{4}\left(u^{\prime}\right)^{2}+\frac{u^{2}}{r^{2}}-\frac{3 \lambda}{8} u^{2}+\frac{\lambda}{16} u^{4}\right) r \mathrm{~d} r
$$

in an appropriately defined admissible space and is, hence, a classical solution of (75a) with $\beta=\sqrt{3}$ and $v(r)=-\sqrt{3} / 2$.

Let $s=-u / \sqrt{6}$. Then (modulo irrelevant additive and multiplicative constants) $\mathcal{E}[u]$ in (79) transforms to

$$
\mathcal{E}[s]=\int_{\rho}^{1}\left(\left(s^{\prime}\right)^{2}+\frac{4 s^{2}}{r^{2}}+\frac{\tilde{\lambda}}{4}\left(2 s^{2}-1\right)^{2}\right) r \mathrm{~d} r,
$$

with $\tilde{\lambda}=3 \lambda / 2=B^{2} R_{\text {out }}^{2} /(2 C L)$, and $s$ is a classical non-negative solution of

$$
s^{\prime \prime}+\frac{s^{\prime}}{r}-\frac{4 s}{r^{2}}=\tilde{\lambda} s\left(2 s^{2}-1\right),
$$

subject to $s(\rho)=s(1)=1 / \sqrt{2}$. Equation (81) is precisely the governing equation for the $2 \mathrm{D}$ defect-free order parameter in (14). We summarize the preceding discussion in the following.

Lemma 2. Let $A=-B^{2} /(3 C)$. Then the 3D LdG Euler-Lagrange equations (69) admit an exact solution of the form (78) where the unknown scalar order parameter $u$ is related to the 2D scalar order parameter $s$ (of the defect-free state) in (11)-(14) by $u=-\sqrt{6} s$ and $\tilde{\lambda}=B^{2} R_{\text {out }}^{2} /(2 C L)$.

i

Next, we discuss the relevance of the local stability result in the 2D setting in Section 3.2, in the full 3D setting.

\footnotetext{
${ }^{\mathrm{i}}$ It is worth saying that solutions of the form (78) exist for other values of $A$ too (see [12] for example) but these solutions have non-constant $\mathbf{E}_{5}$ components for $A \neq-B^{2} /(3 C)$. In other words, we cannot analyze them in terms of a single parameter, such as the function $u(r)$ where $r$ is the $2 \mathrm{D}$ radial distance, as above and the problem necessarily involves two parameters. We work at this special temperature for technical conevenience and for illustrating the main analytical concepts.
} 
The second variation of the $3 \mathrm{D}$ energy (68) about the equilibrium state $\mathbf{Q}^{*}$ is given by

$$
\delta^{2} I\left[\mathbf{Q}^{*}\right]=\int_{\Omega}\left[\frac{1}{2}|\nabla \mathbf{V}|^{2}+\frac{\lambda}{2}\left(\left|\mathbf{Q}^{*}\right|^{2}-1\right)|\mathbf{V}|^{2}+\lambda\left(\mathbf{Q}^{*} \cdot \mathbf{V}\right)^{2}-\lambda \beta \mathbf{Q}^{*} \cdot \mathbf{V}^{2}\right] \mathrm{d} \Omega .
$$

Consider a restricted class of perturbations of the form

$$
\mathbf{V}=v(r, \phi) \mathbf{E}_{1}+w(r, \phi) \mathbf{E}_{2}+\xi(r, \phi) \mathbf{E}_{3}+\eta(r, \phi) \mathbf{E}_{5}
$$

for arbitrary $v, w, \xi, \eta$ such that $v=w=\xi=\eta=0$ on $r=\rho, 1$. This class, albeit restricted, includes four out of the five admissible instabilities and, hence, is a relatively large class of perturbations. Now we set $\beta=\sqrt{3}$ and substitute for $\mathbf{Q}^{*}$ from (78). For perturbations $\mathbf{V}$ of the form (83), the second variation (82) becomes

$$
\begin{aligned}
\delta^{2} I\left[\mathbf{Q}^{*}\right]= & \int_{\Omega}\left[|\nabla v|^{2}+|\nabla w|^{2}+|\nabla \xi|^{2}+\frac{1}{3}|\nabla \eta|^{2}+\frac{1}{r^{2}}\left(4 v^{2}+4 w^{2}+\xi^{2}+v \frac{\partial w}{\partial \phi}-w \frac{\partial v}{\partial \phi}\right)\right. \\
& \left.+\frac{3 \lambda}{2}\left(\left(6 s^{2}-1\right) v^{2}+\left(2 s^{2}-1\right) w^{2}+s(2 s+\sqrt{2}) \xi^{2}+\frac{1}{3}\left(2 s^{2}+1\right) \eta^{2}\right)\right] \mathrm{d} \Omega . \\
\geq & \int_{\Omega}\left[|\nabla v|^{2}+|\nabla w|^{2}+\frac{4}{r^{2}}\left(v^{2}+w^{2}+v \frac{\partial w}{\partial \phi}-w \frac{\partial v}{\partial \phi}\right)\right. \\
& \left.+\tilde{\lambda}\left(6 s^{2}-1\right) v^{2}+\tilde{\lambda}\left(2 s^{2}-1\right) w^{2}\right] \mathrm{d} \Omega .
\end{aligned}
$$

Now the results of Proposition 1 apply directly. The right-hand side of (85) is precisely the functional (20) which was proved to be positive definite provided the inequalities (15a) and $(15 \mathrm{~b})$ are satisfied. Hence, $\mathbf{Q}^{*}$ (in (78)) is indeed locally stable with respect to restricted perturbations of the form (83) in the 3D setting, when the defect-free state is stable in the $2 \mathrm{D}$ sense as discussed in Section 3.2. In other words, any instability in this regime would be induced by perturbations with a $\mathbf{E}_{4}$ component i.e. perturbations which are out of plane with components along the $2 \mathrm{D}$ director $\mathbf{m}$ of the $2 \mathrm{D}$ defect-free state.

Similarly, in Section 3.4, we identify an unstable region of the $(\lambda, \rho)$ plane where there exist admissible perturbation functions $v_{c}(r, \lambda, \rho)$ and $w_{c}(r, \lambda, \rho)$ for which the functional (20) is negative. We can use these functions to define perturbations of the form (83) with $\xi=\eta=0$ i.e. work with $\mathbf{V}=v_{c}(r, \lambda, \rho) \sin 2 \phi \mathbf{E}_{1}+w_{c}(r, \lambda, \rho) \cos 2 \phi \mathbf{E}_{2}$ and evaluate (84) for such a $\mathbf{V}$. The second variation (84) then reduces to $(20)$ and is consequently negative, demonstrating instability in this subset of the $(\lambda, \rho)$-plane.

Lemma 3. The exact solution (78) of the LdG Euler-Lagrange system (69) at the fixed temperature $A=-B^{2} /(3 C)$ is locally stable with respect to perturbations of the form (83) provided the inequalities $(15 \mathrm{a})$ and $(15 \mathrm{~b})$ in $(\tilde{\lambda}, \rho)$ are satisfied. This exact solution is necessarily unstable whenever the 2D defect-free state defined in (11)-(14) is unstable for $\lambda=B^{2} R_{\text {out }}^{2} /(2 C L)$.

Finally, we appeal to the results in [19] to further connect our 2D results to a $3 \mathrm{D}$ setting. In [19], the authors study minimizers of 3D LdG energies of the form (1) on thin 3D geometries of the form $\Omega \times(0, h)$ where $\Omega \in \mathbb{R}^{2}$ and $0<h \ll 1$. They impose uniaxial Dirichlet conditions on the lateral sides and surface energies on the top and bottom; for particular choices of the surface coefficients, the surface energy minimizers are precisely of the form (78). They then compute the $\Gamma$-limits of the $3 \mathrm{D}$ LdG energies, including the surface energies in the limit of $h \rightarrow 0$. They prove that the limiting energy is precisely of the form (1)-(6) on the 2D domain $\Omega$ and the limiting minimizers indeed have the structure given by (78). Thus, a study of critical points of the form (78) is relevant for nematics confined to thin 3D geometries. 


\section{Conclusions}

We study the 2D defect-free "bend" state on a 2D annulus with Dirichlet tangent boundary conditions, in an idealized 2D LdG framework described by symmetric traceless $2 \times 2$ matrices. We define this state in Section 3.1 in terms of an optimal scalar order parameter $s$ defined in (11)-(14). We derive a local stability result in terms of a dimensionless material and temperature-dependent parameter $\lambda$ and the annular aspect ratio $\rho$, and perform a detailed asymptotic analysis that yields a curve in the $(\lambda, \rho)$ plane separating the regions of stability and instability in the $2 \mathrm{D}$ framework. The asymptotic results are of independent interest, particularly since they predict two limiting values of $\lambda$ as $\rho \rightarrow 0$, in two separate distinguished limits. They also predict two critical values of $\lambda$ and $\rho$ such that the defect-free state is stable (within the 2D framework) if $\rho$ is suffiently large (regardless of the value of $\lambda$ ) or if $\lambda$ is sufficiently small (regardless of the value of $\rho$ ). This is consistent with the fact that the $2 \mathrm{D}$ LdG energy has a unique critical point in the limits $\rho \rightarrow 1$ (regardless of $\lambda$ ) and $\lambda \rightarrow 0$ (regardless of $\rho$ ), as can be seen by adapting the arguments in [26]. The agreement between the numerical and the asymptotic results is also very good.

Of course, the relevance of our simplified 2D approach in a 3D framework is not immediately clear, although equivalent 2D LdG models have been used with some success in a batch of experimental and theoretical papers $[14,24,28]$ to model severely confined approximately two-dimensional systems. In $[16,22]$, the authors refer to the $B=0$ case of the LdG energy in (1) as the very low-temperature limit, and the defect-free state defined in Section 3.1 is an exact solution of the LdG Euler-Lagrange equations with $B=0$. We make the connection between the idealized 2D LdG approach and the full 3D LdG setting more precise in Section 4 by relating the defect-free state in (11)-(14) to a radially symmetric $(u, v)$-type solution introduced in [16] at a fixed temperature $A=-B^{2} /(3 C)$. This is not the low-temperature limit but a physically relevant temperature below the critical supercooling temperature $A=0$. The stability results in Sections 3.4 and 3.3 give some information about stability in the $3 \mathrm{D}$ framework. In particular, the stability estimate in Section 3.4 carries over to the stability of the corresponding $3 \mathrm{D}$ radially symmetric solution with respect to a class of restricted perturbations, and the instability results are immediately valid in the 3D framework.

This relation between 2D and 3D solutions is more general. See for example [12,23] (and this list is not exhaustive), where the authors define a well order reconstruction solution (described by a symmetric traceless $3 \times 3$ matrix) which is an exact solution of the $3 \mathrm{D}$ LdG Euler-Lagrange equations in terms of a symmetric, traceless $2 \times 2$ matrix at the special temperature, $A=-B^{2} /(3 C)$ used in Section 4. They show that the properties of the well order reconstruction solution can (to some extent) be analyzed in terms of the solution of a reduced two-dimensional problem, represented by a $2 \times 2$ matrix as in Section 3.1. We conjecture that at the fixed temperature $A=-B^{2} /(3 C)$, we can define a reduced problem in terms of $2 \times 2$ matrices on every regular 2D polygon with Dirichlet conditions and this reduced solution is related to an exact critical point of the 3D LdG energy in (1). We will investigate this conjecture carefully in future work.

\section{Acknowledgements}

We thank Dr. Oliver Dammone for valuable discussions. A. L. is supported by the Engineering and Physical Sciences Research Council (EPSRC) studentship. A. M. is supported 
by an EPSRC Career Acceleration Fellowship EP/J001686/1 and EP/J001686/2, an OCIAM Visiting Fellowship, a UK Fluids Short Research Visit grant and the Keble Advanced Studies Centre. This publication is partly based on work supported by Award No. KUK-C1-013-04, made by King Abdullah University of Science and Technology (KAUST).

\section{References}

[1] J. Alvarado. Biological polymers: confined, bent, and driven. PhD thesis, VU University Amsterdam, 2013.

[2] J. M. Ball. Function spaces for liquid crystals. Nonlinear Function Spaces in Mathematics and Physical Sciences, Dec 2015.

[3] P. J. Barratt and B. R. Duffy. Weak-anchoring effects on a Freedericksz transition in an annulus. Liq. Cryst., 19(1):57-63, 1995.

[4] P. J. Barratt and B. R. Duffy. Freedericksz transitions in nematic liquid crystals in annular geometries. J. Phys. D Appl. Phys., 29(6):1551, 1996.

[5] P. J. Barratt and B. R. Duffy. The effect of splay-bend elasticity on Freedericksz transitions in an annulus. Liq. Crystals, 26(5):743-751, 1999.

[6] P. Bauman, J. Park, and D. Phillips. Analysis of nematic liquid crystals with disclination lines. Arch. Ration. Mech. Anal., 205.

[7] F. Bethuel, H. Brezis, B. D. Coleman, and F. Hélein. Bifurcation analysis of minimizing harmonic maps describing the equilibrium of nematic phases between cylinders. Arch. Ration. Mech. Anal., 118(2):149-168, 1992.

[8] F. Bethuel, H. Brezis, and F. Hélein. Ginzburg-Landau Vortices. Progress in Nonlinear Differential Equations and Their Applications. Birkhäuser Boston, 1994.

[9] G. Bevilacqua and G. Napoli. Periodic splay-twist Fréedericksz transition for nematics confined between two concentric cylinders. Phys. Rev. E, 81:031707, Mar 2010.

[10] P. Biscari and E. G. Virga. Local stability of biaxial nematic phases between two cylinders. Int. J. Nonlinear. Mech., 32(2):337 - 351, 1997.

[11] F. Bisi, E. C. Gartland, R. Rosso, and E. G. Virga. Order reconstruction in frustrated nematic twist cells. Phys. Rev. E, 68:021707, Aug 2003.

[12] G. Canevari, A. Majumdar, and A. Spicer. Order reconstruction for nematics on squares and hexagons: A landau-de gennes study. SIAM Journal on Applied Mathematics, $77(1): 267-293,2017$.

[13] O. J. Dammone. Confinement of Colloidal Liquid Crystals. PhD thesis, University College, University of Oxford, 2013.

[14] A. J. Davidson and N. J. Mottram. Conformal mapping techniques for the modelling of liquid crystal devices. Eur. J. Appl. Math., 23:99-119, 2012. 
[15] P. G. de Gennes and J. Prost. The Physics of Liquid Crystals. International Series of Monographs on Physics. Oxford University Press, second edition, 1998.

[16] G. Di Fratta, J. M. Robbins, V. Slastikov, and A. Zarnescu. Half-integer point defects in the Q-tensor theory of nematic liquid crystals. Journal of Nonlinear Science, 26(1):121140, Feb 2016.

[17] I. C. Gârlea, P. Mulder, J. Alvarado, O. Dammone, D. G. A. L. Aarts, M. P. Lettinga, G. H. Koenderink, and B. M. Mulder. Finite particle size drives defect-mediated domain structures in strongly confined colloidal liquid crystals. Nature Communications, 7:12112, 2016.

[18] D. Golovaty and L. Berlyand. On uniqueness of vector-valued minimizers of the GinzburgLandau functional in annular domains. Calc. Var. Partial Dif., 14(2):213-232, 2002.

[19] D. Golovaty, J. A. Montero, and P. Sternberg. Dimension reduction for the landau-de gennes model in planar nematic thin films. Journal of Nonlinear Science, 25(6):14311451, Dec 2015.

[20] R. Ignat, L. Nguyen, V. Slastikov, and A. Zarnescu. Instability of point defects in a two-dimensional nematic liquid crystal model. In Annales de l'Institut Henri Poincare (C) Non Linear Analysis, volume 33, pages 1131-1152. Elsevier, 2016.

[21] R. Ignat, L. Nguyen, V. Slastikov, and A. Zarnescu. Stability of point defects of degree $p m \frac{1}{2}$ in a two-dimensional nematic liquid crystal model. Calculus of Variations and Partial Differential Equations, 55(5):119, Sep 2016.

[22] G. Kitavtsev, J. M. Robbins, V. Slastikov, and A. Zarnescu. Liquid crystal defects in the Landau-de Gennes theory in two dimensions - Beyond the one-constant approximation. Mathematical Models and Methods in Applied Sciences, 26(14):2769-2808, 2016.

[23] S. Kralj and A. Majumdar. Order reconstruction patterns in nematic liquid crystal wells. Proc. Roy. Soc. A, 470(2169):20140276, 2014.

[24] H. Kusumaatmaja and A. Majumdar. Free energy pathways of a multistable liquid crystal device. Soft Matter, 11:4809-4817, 2015.

[25] X. Lamy. Some properties of the nematic radial hedgehog in the Landau-de Gennes theory. J. Math. Anal. Appl., 397(2):586-594, 2013.

[26] X. Lamy. Bifurcation analysis in a frustrated nematic cell. Journal of Nonlinear Science, 24(6):1197-1230, Dec 2014.

[27] A. H. Lewis, D. G. A. L. Aarts, P. D. Howell, and A. Majumdar. Nematic equilibria on a two-dimensional annulus. Studies in Applied Mathematics, 138(4):438-466, 2017.

[28] C. Luo, A. Majumdar, and R. Erban. Multistability in planar liquid crystal wells. Phys. Rev. E, 85:061702, Jun 2012.

[29] A. Majumdar. Equilibrium order parameters of nematic liquid crystals in the Landau-de Gennes theory. Eur. J. Appl. Math., 21:181-203, 2010. 
[30] A. Majumdar. The Landau-de Gennes theory of nematic liquid crystals: Uniaxiality versus biaxiality. Commun. Pur. Appl. Anal., 11(3):1303-1337, May 2012.

[31] A. Majumdar. The radial-hedgehog solution in Landau-de Gennes' theory for nematic liquid crystals. European Journal of Applied Mathematics, 23:61-97, 2012.

[32] A. Majumdar and A. Zarnescu. Landau-de Gennes theory of nematic liquid crystals: the Oseen-Frank limit and beyond. Arch. Rational Mech. Anal., 196(1):227-280, 2010.

[33] O. V. Manyuhina, K. B. Lawlor, M. C. Marchetti, and M. J. Bowick. Viral nematics in confined geometries. Soft matter, 11(30):6099-6105, 2015.

[34] P. Mironescu. On the stability of radial solutions of the Ginzburg-Landau equation. $J$. Funct. Anal., 130(2):334-344, 1995.

[35] S. Mkaddem and E. C. Gartland Jr. Fine structure of defects in radial nematic droplets. Phys. Rev. E, 62(5):6694, 2000.

[36] N. Mottram and C. Newton. Introduction to Q-tensor theory. Technical report, University of Strathclyde, 2004.

[37] P. Palffy-Muhoray, A. Sparavigna, and A. Strigazzi. Saddle-splay and mechanical instability in nematics confined to a cylindrical annular geometry. Liq. Crystals, 14(4):11431151, 1993.

[38] T.-W. Pan. Existence and multiplicity of radial solutions describing the equilibrium of nematic liquid crystals on annular domains. J. Math. Anal. Appl, 245(1):266-281, 2000.

[39] C. Tsakonas, A. J. Davidson, C. V. Brown, and N. J. Mottram. Multistable alignment states in nematic liquid crystal filled wells. Appl. Phys. Lett., 90(11):111913, 2007.

[40] E. G. Virga. Variational Theories for Liquid Crystals, volume 8. CRC Press, 1995. 